\title{
Categorical Signaling of the Strongest Stimulus by an Inhibitory Midbrain Nucleus
}

\author{
Hannah M. Schryver, ${ }^{1}$ Malgorzata Straka, ${ }^{1}$ and Shreesh P. Mysore ${ }^{1,2}$ \\ ${ }^{1}$ Department of Psychological and Brain Sciences, Johns Hopkins University, Baltimore, Maryland 21218, and ${ }^{2}$ The Solomon H. Snyder Department \\ of Neuroscience, Johns Hopkins University, Baltimore, Maryland 21218
}

The nucleus isthmi pars magnocellularis (Imc), a group of inhibitory neurons in the midbrain tegmentum, is a critical component of the spatial selection network in the vertebrate midbrain. It delivers long-range inhibition among different portions of the space map in the optic tectum (OT), thereby mediating stimulus competition in the OT. Here, we investigate the properties of relative strength-dependent competitive interactions within the Imc, in barn owls of both sexes. We find that when Imc neurons are presented simultaneously with one stimulus inside the receptive field and a second, competing stimulus outside, they exhibit gradual or switch-like response profiles as a function of relative stimulus strength. They do so both when the two stimuli are of the same sensory modality (both visual) or of different sensory modalities (visual and auditory). Moreover, Imc neurons signal the strongest stimulus in a dynamically flexible manner, indicating that Imc responses reflect an online comparison between the strengths of the competing stimuli. Notably, Imc neurons signal the strongest stimulus more categorically, and earlier than the OT. Paired recordings at spatially aligned sites in the Imc and OT reveal that although some properties of stimulus competition, such as the bias of competitive response profiles, are correlated, others such as the steepness of response profiles, are set independently. Our results demonstrate that the Imc is itself an active site of competition, and may be the first site in the midbrain selection network at which stimulus competition is resolved.

Key words: categorization; inhibition; midbrain; selection; spatial attention; superior colliculus

Significance Statement

This work sheds light on the functional properties of a small group of inhibitory neurons in the vertebrate midbrain that play a key part in how the brain selects a target among competitors. A better understanding of the functioning of these neurons is an important building block for the broader understanding of how distracters are suppressed, and of spatial attention and its dysfunction.

\section{Introduction}

For animals operating within complex environments, the ability to select the location of the highest priority stimulus is vital for adaptive behaviors. Stimulus priority is the combination of the physical salience of a stimulus as well as its behavioral relevance, and is computed at several sites in the brain (Fecteau and Munoz, 2006). Among these, the midbrain selection network has been studied for its important role in the control of stimulus

\footnotetext{
Received Jan. 5, 2020; revised Mar. 4, 2020; accepted Apr. 2, 2020

Author contributions: S.P.M., H.M.S., and M.S. designed research; H.M.S. and M.S. performed research; H.M.S. and S.P.M. contributed unpublished reagents/analytic tools; H.M.S. and S.P.M. analyzed data; S.P.M. and H.M.S. wrote the paper.

The authors declare no competing financial interests.

This work was supported by funding from National Institutes of Health Grant R01-EY-027718. We thank James Garmon for assistance in building the equipment necessary for the experiments and Nagaraj Mahajan for help in data collection and analysis.

M. Straka's present address: Paradromics, Inc., Austin, TX 78759

Correspondence should be addressed to Shreesh P. Mysore at shreesh.mysore@jhu.edu.

https://doi.org/10.1523/JNEUROSCI.0042-20.2020

Copyright $\odot 2020$ the authors
}

selection for spatial attention (Knudsen, 2011; Krauzlis et al., 2013). It consists of the superior colliculus [SC (in mammals)] or optic tectum [OT (in nonmammals)], which encodes a topographic map of multisensory and motor space, as well as a spatial map of stimulus priority (Knudsen, 2011; Krauzlis et al., 2013), and satellite brain areas in the midbrain tegmentum that are interconnected with the SC/OT (Graybiel, 1978; Gruberg and Udin, 1978; Sereno and Ulinski, 1987; Jiang et al., 1996; Wang et al., 2004).

Work in nonhuman primates has demonstrated that the intermediate-deep layers of the SC (SCid) are required for the selection of the target of spatial attention amid distractors (McPeek and Keller, 2004; Lovejoy and Krauzlis, 2010). In parallel, work in the avian midbrain has demonstrated that neurons in the intermediate-deep layers of the OT (OTid) signal the highest-priority stimulus categorically: they respond with a high firing rate when the stimulus inside their receptive field (RF) is of highest priority, but are suppressed abruptly to a low firing rate when it is no longer the highest priority (Mysore et al., 2011; Mysore and Knudsen, 2011, 2014). Suppression of the responses 
of SCid/OTid neurons by competing stimuli has been reported in several vertebrate species (Rizzolatti et al., 1974; Frost, 1978; Schellart et al., 1979; McPeek and Keller, 2002, 2004; Felsen and Mainen, 2008; Mysore et al., 2010; Gabay et al., 2013; Duan et al., 2015). The source of such long-range competitive inhibition that underlies the categorical signaling of OTid has been identified to be a group of inhibitory neurons in the vertebrate midbrain tegmentum, called nucleus isthmi pars magnocellularis (Imc; Gruberg and Udin, 1978; Sereno and Ulinski, 1987; Wang et al., 2004). Specifically, it has been shown in birds that inactivation of the Imc abolishes all competitive interactions in the OTid (Marín et al., 2007; Mysore and Knudsen, 2013), as well as in the cholinergic isthmi pars parvocellularis (Ipc; Graybiel, 1978; Jiang et al., 1996; Wang et al., 2006), another key area in the midbrain selection network, which serves as a point-to-point amplifier of activity across the OTid space map (Dudkin and Gruberg, 2003; Marín et al., 2007; Asadollahi and Knudsen, 2016).

Despite the importance of Imc to the signaling of the highestpriority stimulus by the OTid (Marín et al., 2007; Mysore and Knudsen, 2013), its functional properties are not well understood (Yan and Wang, 1986; Wang and Frost, 1991). Recent work in barn owls has revealed the unusual multilobed structure of spatial RFs in the Imc, which has been shown to underlie its combinatorially optimized encoding of visual space (Mahajan and Mysore, 2018). In addition, Imc neurons have been shown to exhibit global inhibitory surrounds that may serve as a substrate for stimulus competition within the Imc (Wang and Frost, 1991; Schryver and Mysore, 2019).

Here, we investigated in detail the properties of multisensory stimulus competition in, and the signaling of the most salient stimulus by, the Imc. Specifically, we examined how the responses of Imc neurons to two competing stimuli changed, as their relative strength (salience) was varied systematically. We compared them with responses of OTid neurons measured either separately, or during simultaneous paired recordings in the two brain areas. We found that Imc neurons display signatures of stimulus competition that are quantitatively different from the OTid on average, and qualitatively distinct from those of individual, spatially aligned OTid sites recorded simultaneously. Together with the finding that the Imc resolves competition earlier than the OT, our results demonstrate that the Imc is not a passive conduit of inhibition to the OT nor does it simply reflect competition occurring there. Rather, the Imc is itself an active site of stimulus competition.

\section{Materials and Methods}

Experimental design and statistical analysis. The central goals of this study were to measure the responses of Imc (and OTid) neurons to a stimulus presented inside the RF, without and with a second stimulus presented simultaneously outside the RF. In addition, with paired recordings in the Imc and OTid following the same protocol, an additional goal was to compare response properties directly at Imc and OTid sites. Each stimulus combination (either single or paired) was repeatedly tested 9-15 times in a randomly interleaved fashion. The data were analyzed, and the results were fit with sigmoids or straight lines, if necessary.

All statistical analyses were conducted within MATLAB. Statistical comparison of the time course of evolution of neural responses between two stimulus conditions was performed on a millisecond-by-millisecond basis using ANOVA. Comparison of the proportions within two distributions (see Fig. 5) was performed using the $\chi^{2}$ test. All other statistical comparisons were performed using nonparametric tests (identified in the text at each instance of a statistical comparison). Correction for multiple comparisons was performed using the Holm-Bonferroni method.
Data shown as $\mathrm{a} \pm \mathrm{b}$ refer to the mean \pm SEM, unless specified otherwise. The ${ }^{\star}$ symbol indicates significance at the 0.05 level (after corrections for multiple comparisons, if applicable).

Neurophysiology. Eleven adult barn owls (Tyto alba; male and female; shared across different studies) were used for electrophysiological recordings. Birds were group housed in an aviary with a $12 \mathrm{~h}$ light/dark cycle. All protocols for animal care and use followed approval by the Johns Hopkins University Institutional Animal Care and Use Committee, and were performed in accordance with National Institutes of Health Guidelines for Care and Use of Laboratory Animals. All experimental and surgical procedures followed previously published methods (Schryver and Mysore, 2019).

Briefly, owls were anesthetized with isoflurane (2\%) and a mixture of nitrous oxide and oxygen (45:55) on experiment days, and head fixed in a sound-attenuating booth. The head fixation was calibrated following published procedures (Knudsen, 1982) such that the dorsolateral tip of the pecten oculi structures within the eyes were positioned at $7^{\circ}$ above the horizon and $\sim 25^{\circ}$ lateral to the vertical midline. Isoflurane administration was ceased after birds were secured. We recorded from single and multiunit sites in the Imc and OT using epoxy-coated tungsten microelectrodes (5 $\mathrm{M} \Omega$ at $1 \mathrm{kHz}$; A-M Systems). Recording sites in the OTid and the Imc were targeted on the basis of stereotaxic coordinates (from prior experiments) and verified on the basis of established neural signatures as described previously (Mysore et al., 2010, 2011; Mysore and Knudsen, 2013; Mahajan and Mysore, 2018; Schryver and Mysore, 2019). For Imc recordings, an electrode was positioned to enter the brain at a medial-leading angle of $5^{\circ}$. At a subset of sites, a second electrode was lowered into OTid to make dual recordings simultaneously. Upon positioning the recording electrodes, nitrous oxide was turned off for the duration of the data collection in some experiments; previous work has established no effect of nitrous oxide tranquilization on neural responses to competition protocols in the midbrain network (Mysore et al., 2011). Spike times were recorded using Tucker-Davis Technologies hardware and custom MATLAB software. Multiunit spike waveforms (OTid and Imc) were sorted into single neurons using the Chronux spike-sorting toolbox (Fee et al., 1996). The quality of the sorted neurons were assessed visually, and were additionally subjected to an $F$ test to determine whether or not each neuron was well isolated from other neurons recorded within the same multiunit site (Mahajan and Mysore, 2018); only well isolated neurons were retained.

Stimuli. Visual stimuli were presented as black, with fixed contrast, expanding looming dots on a gray background on a 65 inch monitor. Looming dot stimuli were used as these reliably evoke strong responses in OT and Imc (Mysore et al., 2010, 2011). The strength of a looming stimulus was defined by its loom speed, with faster loom speeds typically evoking greater responses; the typical range of loom speeds used was 0 to $20 \%$ s. Locations of visual stimuli were defined by double-pole coordinates relative to the midsagittal plane for azimuth or the visual plane for elevation (Knudsen, 1982). Auditory stimuli were presented as broadband noise bursts with equalized amplitudes delivered binaurally through earphones. Sounds were filtered with head-related transfer functions of a standard barn owl (Mysore et al., 2010). Strengths of auditory stimuli were defined by the auditory binaural level (ABL). Visual stimuli were generated using custom MATLAB scripts and psychtoolbox (PTB-3; David, 1997; Pelli, 1997), and auditory stimuli were generated using custom MATLAB scripts and Tucker-Davis Technologies hardware.

Two-dimensional RFs were collected by presenting a stimulus at various azimuthal and elevational locations. These stimuli were either a single looming dot of fixed strength or a stationary dot (radius, $3^{\circ}$ ) moving at a $45^{\circ}$ angle over $3^{\circ}$. For RF measurements, stimuli were presented for five to seven repetitions, with a duration of $250 \mathrm{~ms}$ each and an interstimulus interval of 1000-1500 ms. Spatial locations at which a single stimulus elicited higher firing rates compared with baseline were deemed to constitute the spatial RF of the neuron, and were used to estimate RF extent [half-maximum (max) width] and center (weighted average of RF locations).

Stimulus competition protocols involved the presentation of a visual stimulus (S1) of fixed strength inside the RF, either by itself, or with a second stimulus (either visual or auditory; $\mathrm{S} 2_{\text {vis }}$ or $\mathrm{S} 2_{\text {aud }}$, respectively) of 
varying strengths presented at a distant location (typically, $30^{\circ}$ away from S1). The resulting responses from paired S1 and S2 presentation were collectively called competitor strength-dependent response profiles (or competitor response profiles; CRPs; Mysore et al., 2011). Stimuli were presented for 10-15 repetitions, with a duration of $250 \mathrm{~ms}$ each and an interstimulus interval of $2-3 \mathrm{~s}$.

Data analysis. All analyses were performed using custom MATLAB scripts. Response firing rates were determined by counting the number of spikes over a time window following stimulus onset, converting this count to firing rate $(\mathrm{sp} / \mathrm{s})$, and subtracting the baseline firing rate. The window for computing firing rates was visually estimated to capture evoked responses for each neuron; started, on average, at $120 \mathrm{~ms}$ (115 ms); and had a width, on average, of $170 \mathrm{~ms}$ for Imc (OTid) neurons. Average firing rates and error bars (SEM) were computed from the firing rates across all the repetitions of stimulus presentation, after removing outlier values. Outliers were identified as points that lay outside the range of median $\pm 1.5 \times$ interquartile range of the distribution.

To characterize the responses to the paired presentation of S1 and S2 (i.e., competitor response profiles), we calculated the correlation (Pearson, corrcoef command in MATLAB) as a function of the strength of S2. A significant negative correlation $(p<0.05)$ indicated that responses significantly decreased as S2 strength increased. If a neuron did not exhibit significant negative correlation, it was deemed to exhibit fixed response suppression if the suppression was significant for the majority of S2 values (one-way ANOVA on the firing rates to different competitor strengths followed by post hoc tests against responses to S1 alone, corrected for multiple comparisons using the Holm-Bonferroni correction). The remaining neurons were considered to not show any effect related to the presence of S2.

Negatively correlated competitor response profiles were fit with a standard sigmoidal function, and the parameters of the best fit were determined (Mysore et al., 2011). To obtain reliable estimates of the best fitting sigmoid, any S2 strength for which responses differed nonmonotonically from those of its neighbors, and did so substantially ( $>150 \%$ of the response difference between the neighbors), was omitted from the fitting process and subsequent analyses. We defined the transition range of each competitor response profile as the range of strengths of S2 over which responses decreased from $90 \%$ to $10 \%$ of the max response rate. The half-max of this range was defined as the transition value (i.e., the value of the strength of S2 where responses transitioned from being stronger to weaker than the half-max response). The determination of whether competitor response profiles transitioned abruptly (in a "switch-like" manner) or systematically (in a "gradual" manner) from high to low responses, we adopted previously published conventions (Asadollahi et al., 2010; Mysore et al., 2011), as follows: competitor response profiles with transition ranges that were narrower than $4 \%$, or one-fifth of the physiological range of S2 strengths were considered to be switch-like responses, and others as gradual responses. Similarly, for auditory competitors, competitor response profiles with a transition range of $\leq 9 \mathrm{~dB}$ were considered to be switch like, and the others gradual. Incidentally, these "cutoff" values correspond closely to the dips in the bimodal distributions of transition ranges (Fig. $1 F$; see also Fig. 3D, respectively).

For neurons with negatively correlated competitor response profiles, we also calculated the instantaneous firing rates by first obtaining the poststimulus time histogram (PSTH) of the responses ( $1 \mathrm{~ms}$ time bins), and then smoothing the PSTH with a Gaussian kernel ( $\sigma=12 \mathrm{~ms})$. For each neuron, the instantaneous firing rates to the paired presentation of S1 and S2 (as well as to S1 alone) were normalized by the peak of the average instantaneous firing rate to $\mathrm{S} 1$ alone.

The pooled population responses in Figure 2 (see also Figs. 5, 6) were obtained by binning relative strengths (S2-S1) into five bins and combining the responses of all the neurons within each bin (Mysore et al., 2011). This was done separately for OTid and Imc neurons, and separately for neurons with gradual and switch-like competitor response profiles. The time course of response suppression by S2 was determined (within each relative strength bin) by performing a millisecond-by-millisecond ANOVA, comparing the pooled instantaneous firing rates to S1 alone versus to S1 and S2 presented together (Sasikumar et al., 2018).
The time to suppression was defined as the first millisecond at which the $p$ value of the ANOVA comparison dropped to $<0.05$, remained at $<0.05$ for the next $25 \mathrm{~ms}$, and reached 0.01 at least once in that period (Mysore et al., 2011).

Discriminability (D-prime) between responses to two stimulus conditions was computed as the difference in mean responses over the square root of the average of the variances.

For comparison of results from dual simultaneous recordings in the Imc and OTid, we performed all analyses on data from pairs of multiunit sites, rather than on data from pairs of single neurons sorted from these multiunit sites. This is because there was no rational way of establishing which specific neurons sorted from the Imc site ought to be paired with which neurons in the OTid site; comparing all possible pairs would violate the assumption of statistical independence across samples. Notably, because multiunit sites are indeed activated as a whole upon presentation of stimuli, the comparison of Imc and OTid site responses was appropriate and meaningful.

\section{Results}

\section{Switch-like and gradual response profiles in the Imc to competing visual stimuli}

To examine relative strength-dependent stimulus competition in the Imc, we recorded extracellular responses of Imc neurons in the barn owl, using a previously published competition protocol (Materials and Methods; Asadollahi et al., 2011; Mysore et al., 2011; Fig. 1A). We presented a visual stimulus (S1) of fixed strength (loom speed; Materials and Methods) within the RF of a recorded neuron, and measured responses when a second, competing visual stimulus (S2) of varying strengths was presented far outside the RF ( $>30^{\circ}$ away; Fig. $1 A$; Mysore et al., 2011). S1 and S2 were presented simultaneously and for the same duration (250 ms; Materials and Methods). The responses obtained to the paired presentation of S1 and S2 using this protocol, collectively called competitor strength-dependent response profiles (or competitor response profiles; Fig. 1B,D, bottom panels; Mysore et al., 2011), were compared with the responses to $S 1$ presented alone (Fig. 1B,D, top panels); the two types of trials were interleaved randomly.

The majority of the recorded Imc neurons (66 of 78) exhibited competitor response profiles that were negatively correlated with the strength of S2 (Fig. $1 B-E ; p<0.05$, Pearson correlation test; Materials and Methods). Of the remaining, a small subset showed competitor response profiles with fixed response suppression, independent of the strength of S2 (1 of 78; Materials and methods), and the rest were not affected by S2 (11 of 78; Materials and Methods).

Further examination of the negatively correlated competitor response profiles revealed two distinct patterns of suppression based on how abruptly the responses transitioned from the maximum to the minimum value. In one set of competitor response profiles, the majority of response suppression was expressed over a narrow range of S2 strengths, and in the other, the response suppression increased in a graded, systematic way as a function of S2 strength. To quantify the abruptness of the response transition, we defined the competitor response profile transition range as the range of S2 strengths over which the responses dropped from $90 \%$ to $10 \%$ of the maximum response (Materials and Methods). Following a previously published convention (Mysore et al., 2011; Materials and Methods), competitor response profiles with transition ranges narrower than one-fifth the nominal range of S2 strengths $\left(4^{\circ} / \mathrm{s}\right)$, were referred to as being switch-like (Fig. $1 B, C$ ), while those with transition ranges broader than $4 \%$ were referred to as being gradual (Fig. 1D,E). 
A

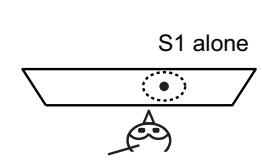

Switch-like

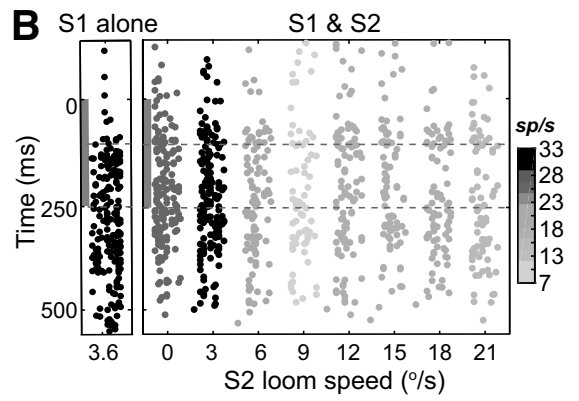

C

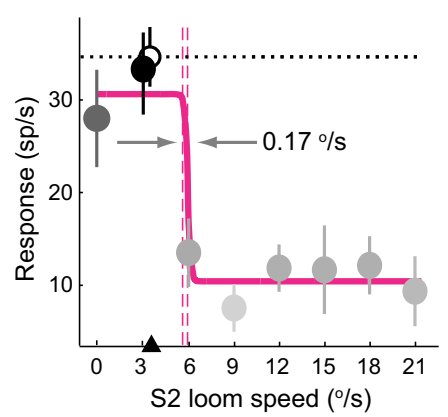

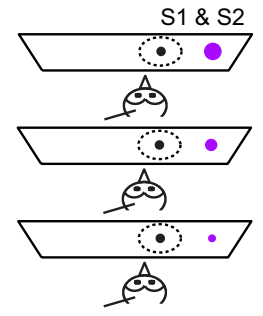

Gradual

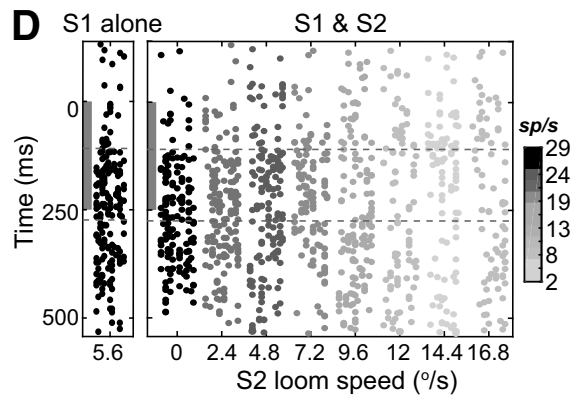

$\mathbf{E}$

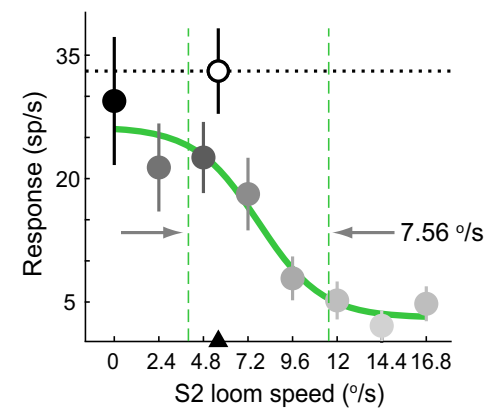

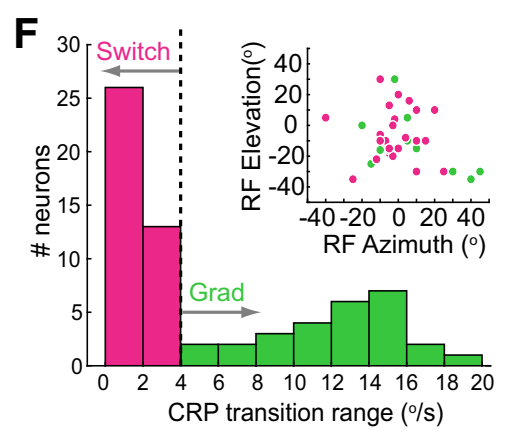

Figure 1. Switch-like and gradual response suppression of Imc neurons by a competing stimulus. $A$, Schematic of experimental setup and stimulus protocol for measuring competitor strength-dependent response profiles (CRPs). Quadrilateral, Tangent monitor; dashed oval, RF of recorded Ime neuron; black dot; S1 visual looming stimulus; magenta dots, S2 visual looming stimulus. Sizes of dots denote loom speeds. $\boldsymbol{B}, \boldsymbol{C}$, Responses of an example $\mathrm{Imc}$ neuron with switch-like competitor response profile (data in magenta). $\boldsymbol{B}$, Rasters of spike responses to $\mathrm{S} 1$ alone (left; loom speed $=3.6^{\circ} / \mathrm{s}$ ) and to the paired stimuli (right) showing an abrupt increase in suppression with increasing $\$ 2$ strength. Shaded box along the $y$-axis represents stimulus duration $(250 \mathrm{~ms})$, and dashed lines denote the time window $(100-250 \mathrm{~ms})$ during which response firing rates were calculated. $\boldsymbol{C}$, Response firing rates corresponding to rasters in $\boldsymbol{B}$. Open circle and horizontal dotted line response firing rate to $\mathrm{S} 1$ alone (mean $\pm \mathrm{SEM}$ ); filled circles with graded grey shading, response firing rates to paired presentation of S1 and S2 (i.e., (RP; mean \pm SEM; darker colors indicating higher firing rates). Correlation coefficient of responses versus $\mathrm{S} 2$ strength $=$ $-0.74\left(p=0.04\right.$, Pearson correlation test). Solid line, Best fitting sigmoid to the competitor response profile $\left(r^{2}=0.95\right)$; vertical dashed lines, transition range of this competitor response profile $(0.17 \% \mathrm{~s}$; Materials and Methods); black arrowhead, strength of $S 1\left(3.6^{\circ} / \mathrm{s}\right)$. D, E, Responses of an example $\operatorname{Imc}$ neuron with gradual competitor response profile (data in green). Conventions are as in $\boldsymbol{B}$ and $\boldsymbol{C}$. Loom speed of $\mathrm{S} 1=5.6 \%$, correlation coefficient of responses versus $S 2$ strength $=-0.94$, $p=0.0002$, Pearson correlation test; $r^{2}=0.99$ for the best fitting sigmoid; transition range $=5.75^{\circ} / \mathrm{s}$. $\boldsymbol{F}$, Histogram of transition ranges of competitor response profiles that exhibited a negative correlation with the strength of $S 2$ ( $n=66$ neurons $/ 78$ total). Vertical line: "cutoff" transition range of $4 \%$ (see "Materials and Methods"). The median strength of $\mathrm{S} 1$ was $7 \%$ with $95 \% \mathrm{Cl}$ of $6.3^{\circ} / \mathrm{s}, 7.7^{\circ} / \mathrm{s}$; median distance of $\mathrm{S} 2$ from $\mathrm{S} 1=43^{\circ}$ with $95 \% \mathrm{Cl}$ of $40^{\circ} 46^{\circ}$. Inset, RF locations (in double-pole coordinates) of Imc neurons at which competitor response profiles were recorded; colors correspond to whether the neurons had switch-like (magenta) or gradual (green) competitor response profiles.

Across the population of $66 \mathrm{Imc}$ neurons that exhibited negatively correlated competitor response profiles, the majority (39 of $66 ; 59 \%$ ) of neurons exhibited switch-like responses, and the rest (27/66; 40\%) exhibited gradual competitor response profiles (Fig. 1F). Switch-like and gradual competitor response profiles were recorded at neurons both encoding for locations within the frontal portion of visual space (within $\pm 15^{\circ}$ in azimuth and elevation), as well as in the more peripheral portion (Fig. $1 F$, inset), indicating that switch-like or gradual modulation of response suppression did not depend on the spatial location of the RF.

\section{Time course of stimulus competition} in the Imc

The time course of response suppression was different between Imc neurons with gradual versus switch-like competitor response profiles. For each neuron with a gradual competitor response profile, we first calculated the instantaneous firing rate responses to $\mathrm{S} 1$ alone, and to the paired presentation of S1 and S2 for every strength of S2 (Materials and Methods). We then binned the relative competitor strength (S2-S1) values into five bins (Fig. $2 A-H$, columns), and, for each bin, grouped across neurons the normalized instantaneous firing rate responses to $\mathrm{S} 1$ alone, and separately, to paired S1 and S2 (Fig. 2, compare $A, B$; Materials and Methods). We quantified the emergence of response suppression for each relative strength bin by comparing the pooled responses to $S 1$ and the pooled responses to paired $\mathrm{S} 1$ and $\mathrm{S} 2$ (Fig. 2C, gray vs green, respectively) using a millisecond-by-millisecond running ANOVA procedure (Fig. 2D; Materials and Methods). The time to suppression was defined as the first instant at which the responses diverged significantly (Fig. 2D; dashed vertical arrows; Materials and Methods).

We found that among the neurons with gradual competitor response profiles, there was a systematic reduction in the time to suppression as a function of relative strength, as follows: from $253 \mathrm{~ms}$ at relative strength of $-7 \%$ s to $124 \mathrm{~ms}$ at relative strength of $+10 \% \mathrm{~s}$ (Fig. 2I, green data; slope $=-6.6 ; 95 \%$ $\mathrm{CI},-12.4,-0.72$; linear regression). We repeated the above analysis for neurons with switch-like competitor response profiles and found that the times to suppression also decreased as a function of relative strength (from 370 to $91 \mathrm{~ms}$ ), but with a much steeper slope than for 
A Gradual CRPs
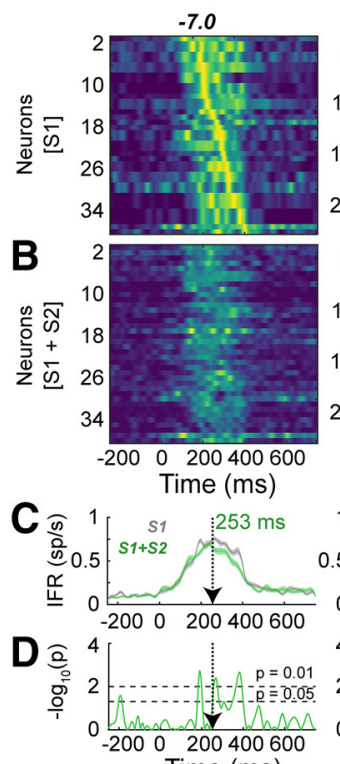
Time (ms)

\section{E Switch-like CRPs}

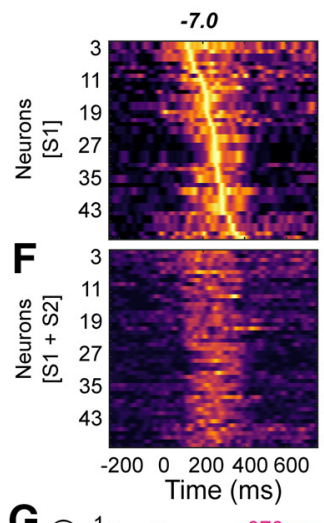

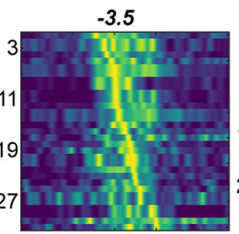

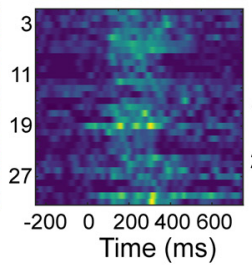

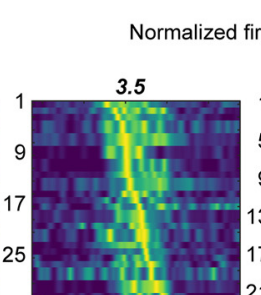

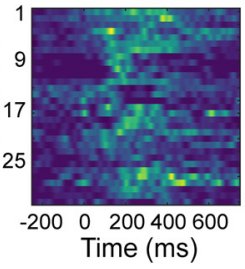

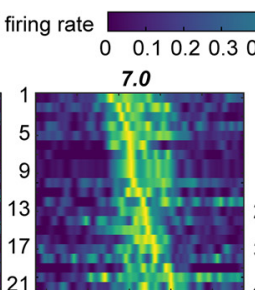

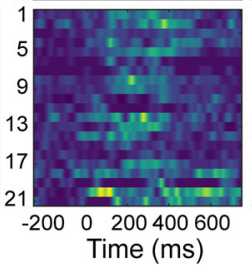

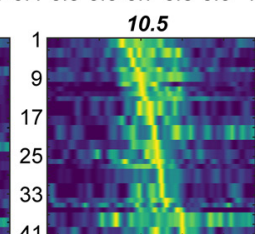

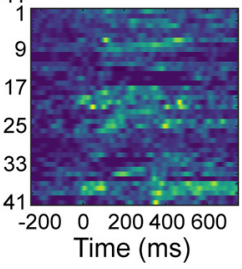

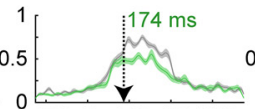
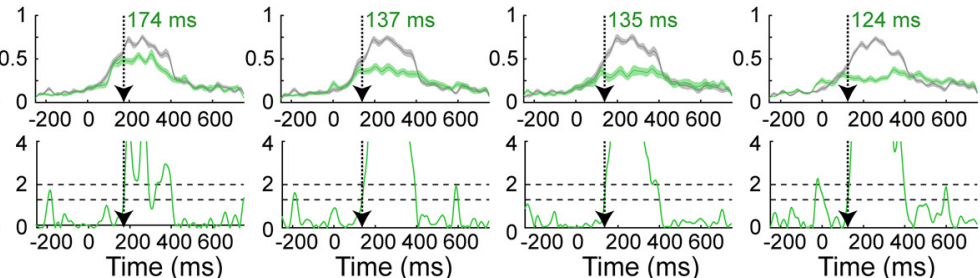

Normalized firing rate

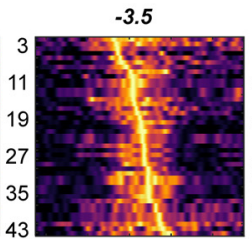

3.5

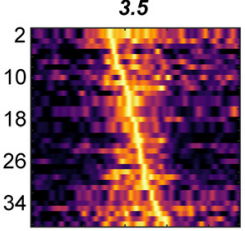

$\begin{array}{lllllllllllll}0 & 0.1 & 0.2 & 0.3 & 0.4 & 0.5 & 0.6 & 0.7 & 0.8 & 0.9 & 1\end{array}$
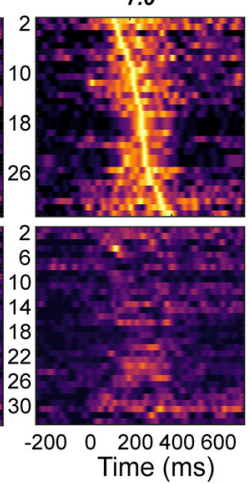
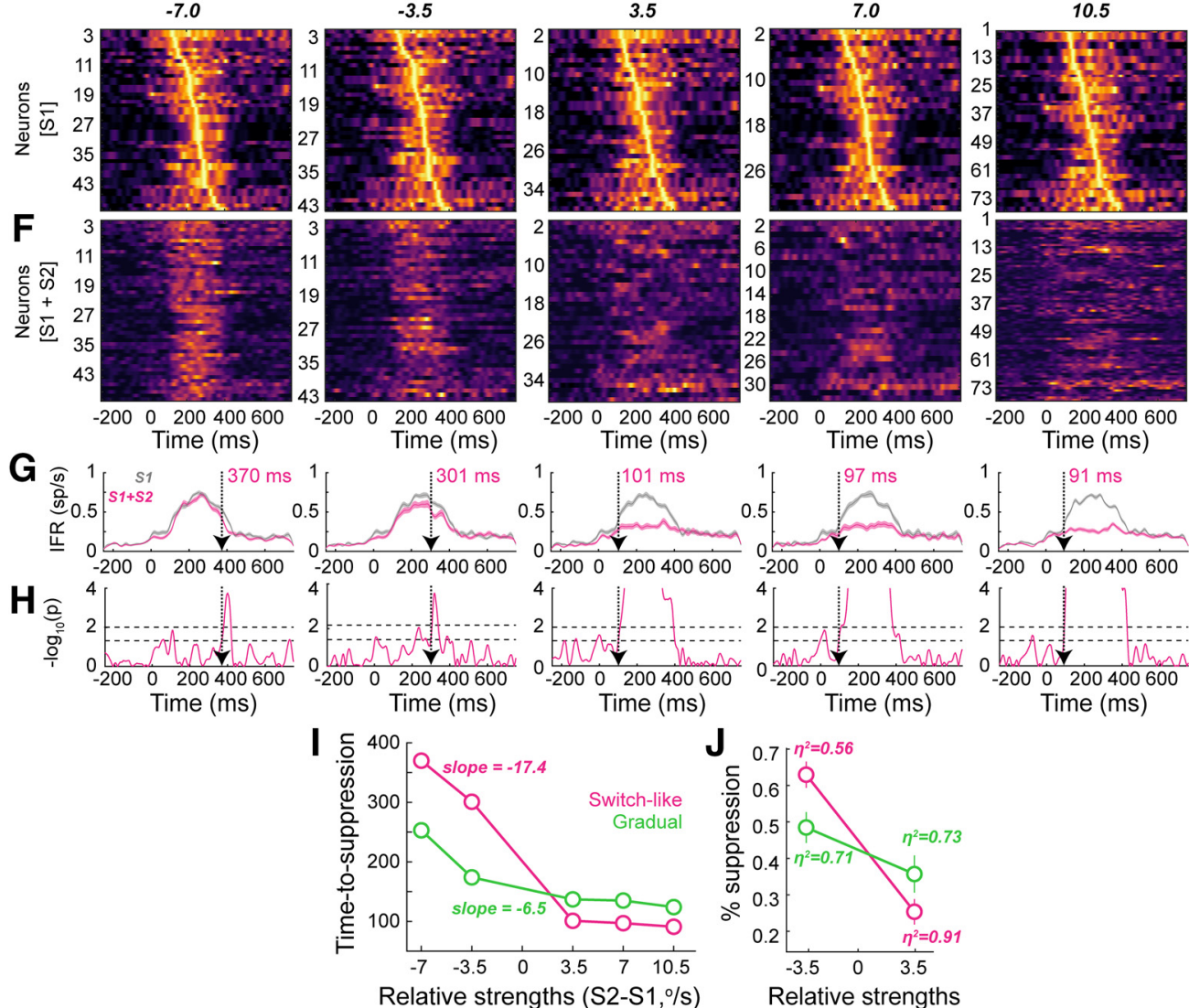

Figure 2. Time course of response suppression for Imc neurons with switch-like versus gradual competitor response profiles (CRPs). $\boldsymbol{A}-\boldsymbol{D}$, Analysis of response time courses of Imc neurons with gradual competitor response profiles; columns, responses binned by the relative strength of S1 and S2 (S2-S1). $\boldsymbol{A}, \boldsymbol{B}$, Instantaneous firing rates (IFRs) of neurons to S1 alone ( $\boldsymbol{A}$ ) or to S1 and S2 presented together $(\boldsymbol{B})$, computed by smoothing PSTHs (1 ms time bins) with a Gaussian kernel (SD =12 ms; Materials and Methods). For each neuron, instantaneous firing rates are normalized by the peak firing rate of that neuron to S1 alone. Neurons in $\boldsymbol{A}$ are sorted by the half-peak firing rate; neurons in $\boldsymbol{B}$ are in the same order as in $\boldsymbol{A}$. $\boldsymbol{C}$, Pooled average firing rates to S1 alone (gray) or to S1 and S2 (green) of all neurons within each bin. Translucent bands indicate SEM. D, Time course of $p$ values obtained by performing ANOVA between the responses to S1 versus to S1 and S2 at each millisecond. Vertical dashed arrows (and colored text), time to suppression (defined as the first instant at which responses to paired S1 and S2 diverge significantly from responses to S1 alone; Materials and Methods); horizontal dashed lines, $p$ value thresholds used in determining time to suppression (bottom line, $p=0.05$; top line, $p=0.01$. $\boldsymbol{E}-\boldsymbol{H}$, Same as $\boldsymbol{A}-\boldsymbol{D}$, but for Imc neurons with switch-like competitor response profiles. I, Comparison of time to suppression for neurons with switch-like (magenta) versus gradual competitor response profiles (green) $J$, Plot of average amount of response suppression ( \pm SEM) for switch-like versus gradual neurons for the two relative strength bins on either side of the selection boundary. Text reports the effect size ( $\eta^{2}$ ). 
A
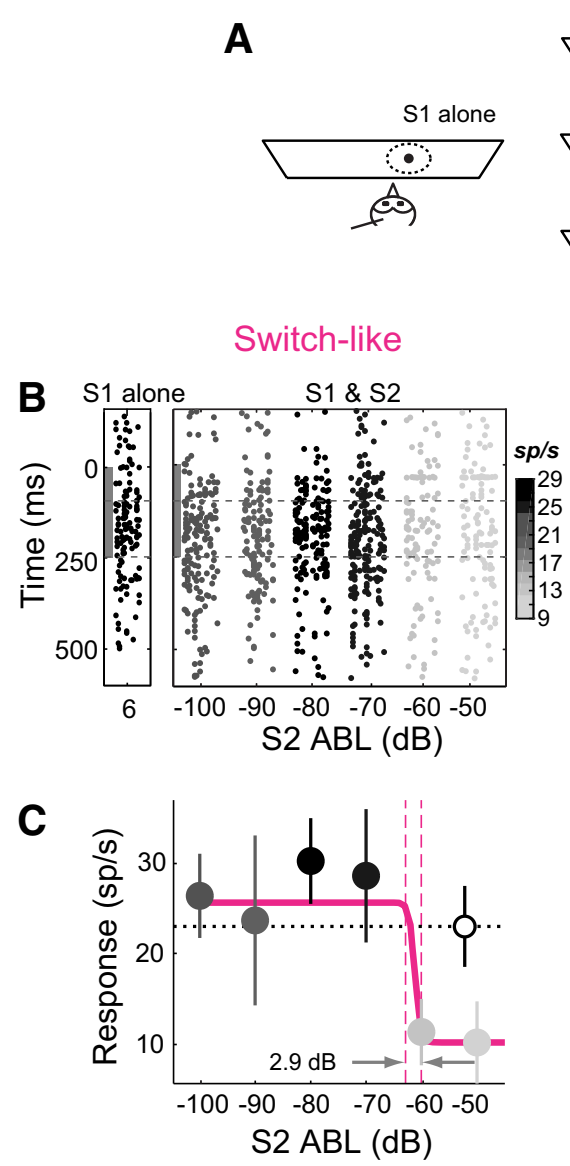
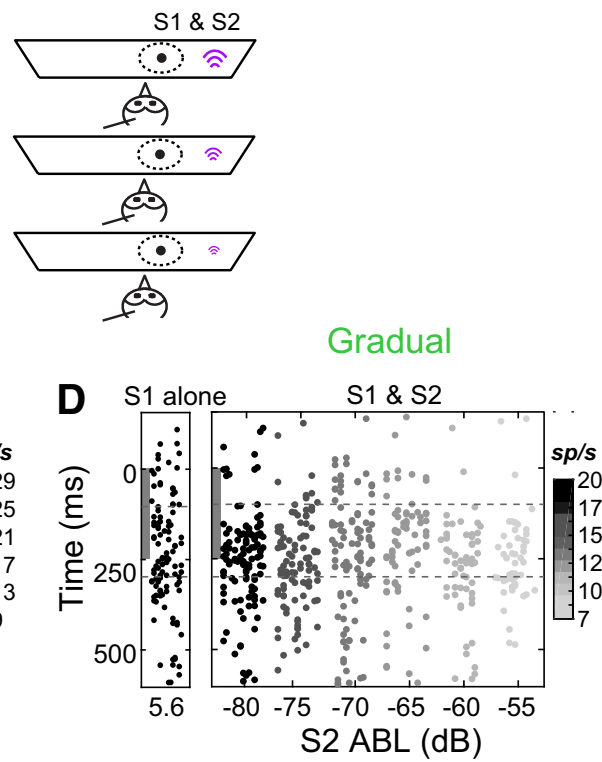

E

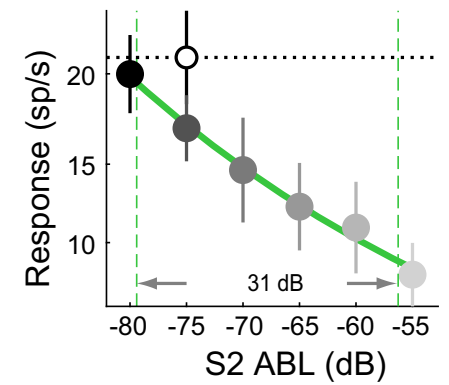

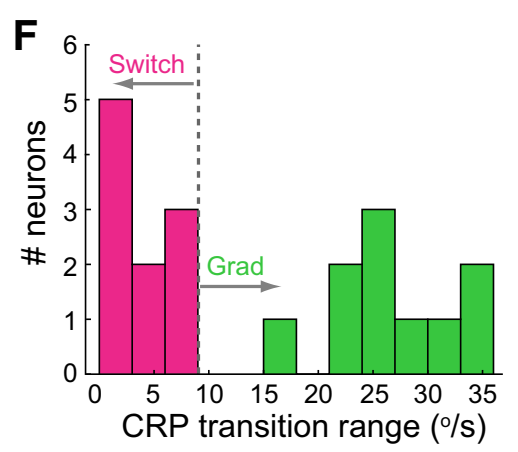

Figure 3. Switch-like and gradual response suppression of Imc neurons by an auditory competitor. $A$, Schematic of experimental setup and stimulus protocol for measuring auditory competitor response profiles (conventions are as in Fig. 1A). Black dot, S1 visual looming stimulus; magenta sound symbol, S2 aud; size of symbol represents binaural intensity of $\mathrm{SZ}_{\text {aud }}$. B, C, Switch-like auditory competitor response profile measured at an example Imc neuron. $\boldsymbol{B}$, Response rasters. Dashed lines, time window (100-250 ms) during which response firing rates were calculated. S1 loom speed $=6 \%$. C, Spike counts. Correlation coefficient of versus $S 2_{\text {aud }}$ strength $=-0.82\left(p=0.04\right.$, Pearson correlation test); $r^{2}=0.98$ for the best fitting sigmoid, transition range $=2.9 \mathrm{~dB}$. All other conventions are as in Figure $1, B$ and $\boldsymbol{C}$. D, $\boldsymbol{E}$, Gradual auditory competitor response profile measured at an example Imc neuron. $\boldsymbol{D}$, Rasters. Dashed lines, Time window (100-300 ms) during which response firing rates were calculated. $S 1$ loom speed $=5.6 \%$. $\boldsymbol{E}$, Spike counts. Correlation coefficient of responses versus $S 2_{\text {aud }}$ strength $=0.99(p=0.00008$, Pearson correlation test $), r^{2}=0.99$ for the best fitting sigmoid, transition range $=31 \mathrm{~dB}$. All other conventions as in Figure 1, D and $E$. $\boldsymbol{F}$, Histogram of transition ranges of competitor response profiles recorded in Imc that exhibited a negative correlation with the strength of $S 2_{\text {aud }}$ ( $n=20$ neurons $/ 35$ total). Vertical line: "cutoff" transition range of $9 \mathrm{~dB}$ (Materials and Methods). The median strength of $\mathrm{S} 1$ was $5.6 \%$; $95 \% \mathrm{Cl}: 5.1^{\circ} / \mathrm{s}, 6.1^{\circ} / \mathrm{s}$. Grad, Gradual; Switch, switch-like.

neurons with gradual competitor response profile (Fig. 2I, magenta data; slope $=-17.4 ; 95 \% \mathrm{CI},-28.1,-6.8$; linear regression). There was, however, no systematic difference in the response latency of neurons with gradual versus switch-like competitor response profiles (latency of response to S1 alone: gradual: median = $136 \mathrm{~ms} ; 95 \%$ CI, 113, 159; switch-like: median $=110 \mathrm{~ms}$; 95\% CI, 95, $125 ; p=0.11$; sign test).

Notably, the times to suppression for switch-like competitor response profiles exhibited a large, abrupt change across the relative strength of zero (i.e., the "selection boundary"), compared with gradual competitor response profiles (Fig. 2I; switch-like, $200 \mathrm{~ms}$ change from 301 to $101 \mathrm{~ms}$; gradual, $37 \mathrm{~ms}$ change from 174 to $137 \mathrm{~ms}$ ). This large change was accompanied by a "flip" in the values of time to suppression for switch-like versus gradual response profiles across the selection boundary, as follows: longer times to suppression for switch-like competitor response profiles when S2 $<$ S1 (Fig. 2I, magenta vs green data at negative relative strength values), but shorter times to suppression for switch-like competitor response profiles when S2 $>$ S1 (Fig. 2I, magenta vs green data at positive relative strength values). This flip was accounted for by intrinsic differences in the properties of switch-like versus gradual competitor response profiles in the Imc and, specifically, by the relative magnitudes of suppression. Neurons with switch-like response profiles showed weak suppression when S2 $<$ S1, weaker than for gradual response profiles, predicting later times to suppression, but showed strong suppression when $\mathrm{S} 2>\mathrm{S} 1$, stronger than for gradual response profiles (Fig. 2J), predicting earlier times to suppression.

\section{Multisensory stimulus competition in the Imc}

The occurrence of gradual and switch-like competitor response profiles was not restricted to just the visual sensory modality. We measured "auditory" competitor response profiles using a visual S1 (of fixed strength) and an $S_{2}$ aud (of varying strengths). $S 2_{\text {aud }}$ stimuli were broadband noise bursts, and $\mathrm{S}_{\text {aud }}$ "strength" was varied by changing the binaural sound level (Materials and Methods).

We recorded auditory competitor response profiles of $35 \mathrm{Imc}$ neurons, and, of these, 20 exhibited competitor response profiles that were negatively correlated with the strength of $S 2_{\text {aud }}$ (Materials and Methods; $p<0.05$, Pearson correlation test). Further examination of the responses of these neurons revealed two distinct patterns of suppression as a function of the strength of $\mathrm{S2}_{\text {aud }}$ (gradual or switch like; Fig. 3B-E). Switch-like auditory competitor response profiles were defined as those for which the transition range was narrower than $9 \mathrm{~dB}$ (one-fifth the full range of $\mathrm{S}_{\text {aud }}$ strengths; just as in the case of visual competitor response profiles, and 
consistent with previously published literature; Asadollahi et al., 2010; Mysore et al., 2011). Of the 20 neurons with correlated competitor response profiles, 10 were found to be gradual and the rest, switch-like (Fig. 3D). Thus, consistent with the role of Imc in enabling within-sensory as well as cross-sensory stimulus competition in the OTid (Mysore and Knudsen, 2013), Imc neurons themselves exhibit signatures of multisensory stimulus competition (Schryver and Mysore, 2019).

Imc signals the strongest stimulus accurately and flexibly The observation of abrupt response suppression in switch-like competitor response profiles led us to ask whether the strength of S2 at which the transition occurred from high to low response values was meaningful. Because this transition was also well defined (albeit less so) in gradual competitor response profiles, we asked this question more generally of switch-like as well as gradual competitor response profiles in the Imc. Specifically, we wondered whether the strength of S2 at which the transition occurred was related to the (fixed) strength of the stimulus inside the RF, S1 (because this comparison is only meaningful when both stimuli are of the same sensory modality, we restricted our analysis to visual competitor response profiles). To this end, we first defined as the competitor response profile "transition value" the strength of S2 at which the responses were half-way between the maximum and minimum values, and quantified it as the midpoint of the transition range (Fig. 4A; Materials and Methods). We then compared the transition value of each competitor response profile to the strength of S1 used to measure the competitor response profile, and defined this difference as the competitor response profile "bias" (bias = transition value strength of S1; Fig. 4A).

Across the population of Imc neurons with correlated visual competitor response profiles $(n=66)$, we found neurons with a range of competitor response profile biases (Fig. $4 B$ ). Some had a negative bias, indicating that these competitor response profiles transitioned from a high to a low value when S2 was less than S1, and others had a positive bias, indicating that those competitor response profiles transitioned from a high to a low value when $\mathrm{S} 2$ was greater than S1. However, across the population, the competitor response profile bias was distributed around zero (Fig. $4 B$; median competitor response profile bias $=-0.52, p=0.39$; sign test against 0 ). This indicated that, on average, Imc neurons responded at a high level when S1 (RF stimulus) was the strongest stimulus, but transitioned to responding at a low level when S1 was no longer the strongest stimulus (i.e., just when S2 exceeded S1 in strength). This was true separately both for switch-like as well as for gradual competitor response profiles (Fig. $4 C$, top and bottom panels, respectively; switch-like: median bias $=-0.68 ; p=0.52$; sign test against $0 ; n=39$ neurons; gradual: median bias $=-0.36 ; p=0.7$; sign test against $0 ; n=27$ neurons).

These results indicated that Imc may signal the strongest of the competing stimuli without any bias (i.e., "accurately"), and suggested the interesting possibility that transition values of Imc competitor response profiles are not fixed quantities, but are coupled "flexibly" to the strength of S1. To test this hypothesis that competitor response profile transition values depend on the strength of S1, we measured two competitor response profiles for each of a subset of Imc neurons. One competitor response profile was measured with a weaker S1, another with a stronger S1 $(\mathrm{S} 1+6 \% \mathrm{~s})$, with $\mathrm{S} 2$ varying over the same range of loom speeds in both cases (Figure 4D,G; Mysore et al., 2011). The stimuli corresponding to the two competitor response profiles were presented in a randomly interleaved manner. We found that the competitor response profile transition value shifted with S1 strength in the predicted way: a stronger S1 produced a rightshifted competitor response profile (Fig. $4 E, F$, data from example neuron with switch-like competitor response profile showing a right shift in transition value; Fig. 4H,I, data from example neuron with gradual competitor response profile showing a right shift). Across the population of tested neurons, we found that the magnitude of the shift in competitor response profile transition value matched, on average, the magnitude of the change in S1 strength [Fig. $4 H$; median shift ratio (shift in transition values/ change in S1 strength) $=0.89 ; p=0.26$; sign test against $1 ; n=27$ neurons].

Together, these results established that Imc neurons report dynamically an online comparison between the strengths of the two competing stimuli. They signal accurately (with average bias indistinguishable from 0 ) the strongest of the two competing stimuli, and do so flexibly (with transition values coupled to the strength of S1).

\section{Comparison of signatures of stimulus competition in the Imc and OTid}

The findings of switch-like and gradual competitor response profiles in the Imc, as well as accurate and flexible signaling of the strongest stimulus, parallel previous findings in two other key nuclei in the midbrain selection network, namely the OTid (Mysore et al., 2011; Mysore and Knudsen, 2011, 2014) and the cholinergic Ipc (Asadollahi et al., 2010). These nuclei are interconnected as follows: Imc receives focal input from layer 10 of the OT $\left(\mathrm{OT}_{10}\right)$, but projects back broadly across the OTid as well as the Ipc (Wang et al., 2004), and Ipc receives focal input from $\mathrm{OT}_{10}$ and projects back focally to the OT (Wang et al., 2006), serving to amplify OTid responses (Marín et al., 2007; Asadollahi and Knudsen, 2016). Additionally, Imc is the source of competitive inhibition in the OTid and Ipc, as follows: focal inactivation of Imc abolishes competitive interactions in both areas (Marín et al., 2007; Mysore and Knudsen, 2013). Considering this interconnectedness, we were interested in whether the signatures of stimulus competition in the Imc simply reflect computations occurring previously (for instance, in the OT), or if the Imc itself serves as an active site at which computations related to stimulus competition occur. We focused on the comparison between Imc and OTid (rather than Ipc as well) because the OTid is the "output" hub of the midbrain selection network, involved in controlling behavior as well as relaying information from the midbrain to cortical areas (Shipp, 2004; Knudsen, 2018).

To address this question, we compared the metrics of stimulus competition in Imc and OTid. To minimize the impact of any idiosyncratic differences in spike sorting, selection of count windows, or other analysis choices on this comparison, we measured competitor response profiles in the OTid as well (Materials and Methods; Mysore et al., 2011).

First, we compared the accuracy with which Imc versus OTid neurons, on average, signal the strongest stimulus. We found that the competitor response profile bias distributions were not distinguishable (Fig. $5 A$; red vs blue data; $p=1.0$; sign test), indicating that Imc and OTid neurons signaled the strongest stimulus with comparable accuracy.

Next, we compared the relative proportions of neurons that exhibited switch-like, gradual, or uncorrelated competitor response profiles in the Imc versus OTid (Fig. $5 B$ ). The proportions were different between Imc and OTid (Imc: $n=78$ neurons; $50 \%$ switch like; $34.6 \%$ gradual; and $15.4 \%$ uncorrelated competitor response profiles; OTid: $n=53$ neurons; $37.7 \%$ switch like; 
A

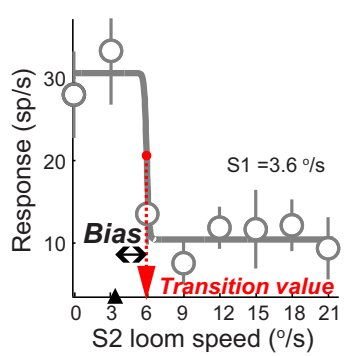

B

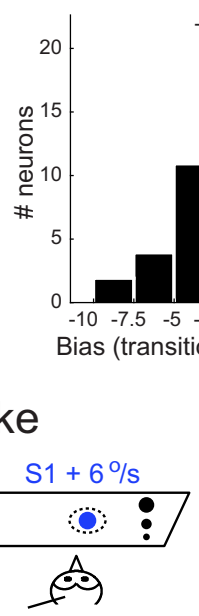

E
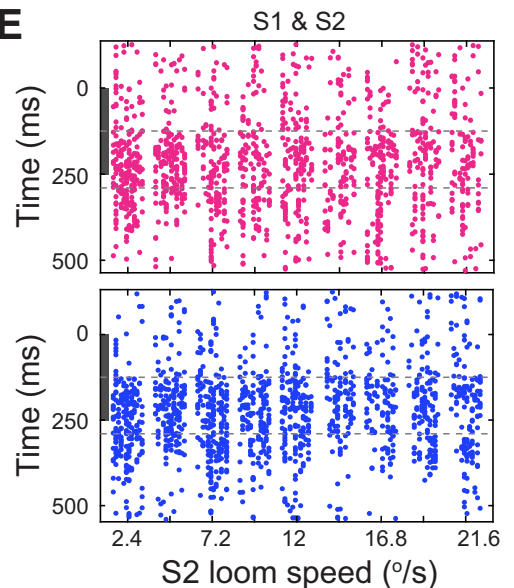

$\mathbf{F}$

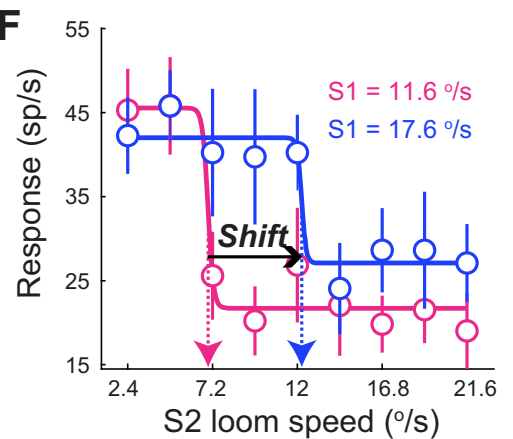

C

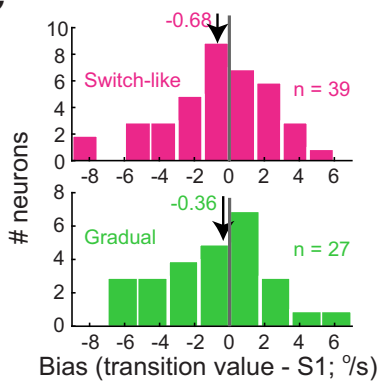

G

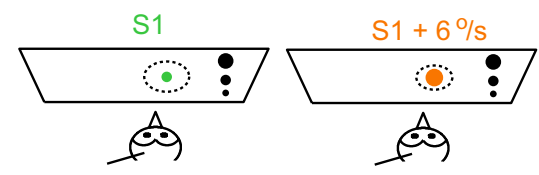

H
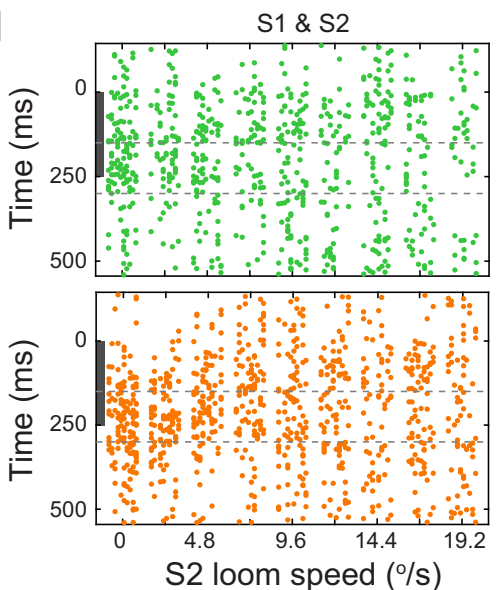

I

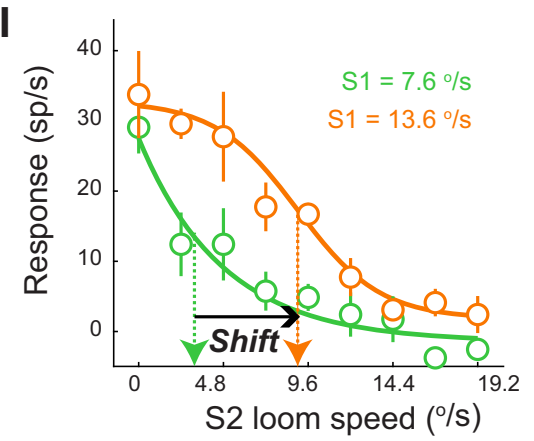

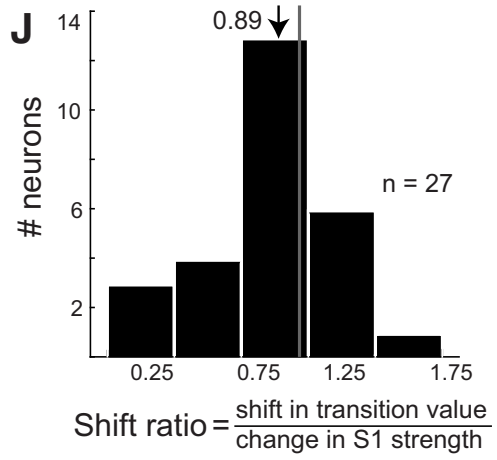

Figure 4. Dependence of transition value of competitor response profile on S1 strength for Imc neurons. A, Definition of transition value of competitor response profile and competitor response profile bias for switch-like visual competitor response profile in Figure $1 C$ (see Materials and Methods). $S 1=3.6 \%$ (black triangle); transition value $=5.9^{\circ} / \mathrm{s}$; bias $=2.3 \%$. $B$, Distribution of competitor response profile bias for visual competitor response profiles; $n=66 \mathrm{Imc}$ neurons. Median $=-0.52, p=0.39$; sign versus 0 .
$30.2 \%$ gradual; and $32.1 \%$ uncorrelated competitor response profiles; $\chi^{2}$ statistic $=5.21, p=0.02$, followed by Holm-Bonferroni correction for multiple comparisons; see below). Indeed, the proportions of competitor response profile types measured in the OTid in this study were not significantly different from those previously published, confirming the veracity of our results (OTid published data; Mysore et al., 2011; $n=169$ neurons; $30.2 \%$ switch like; $33.1 \%$ gradual; and $36.7 \%$ uncorrelated competitor response profiles; $\chi^{2}$ statistic $=-90.96$; $p=1.0$ between measured and published OTid followed by HolmBonferroni correction for multiple comparisons).

The larger fraction of switch-like competitor response profiles in the Imc than the OTid (50\% Imc vs $37.7 \%$ OTid) suggested that Imc ensembles may be able to signal the strongest stimulus more categorically than the OTid (Mysore and Knudsen, 2011) or, in other words, that the signaling of the strongest stimulus may be more "precise" in the Imc (Fig. 5C). To test this directly, we quantified a metric of categorical signaling: the discriminability $\left(\mathrm{d}^{\prime}\right)$ across the selection

$\leftarrow$

C, Distributions of biases of competitor response profiles, separately for Imc neurons with switch-like competitor response profiles (top) and gradual competitor response profiles (bottom). Switch-like: median bias = $-0.68, p=0.52$, sign test against 0 followed by Holm-Bonferroni correction for multiple comparisons, $n=39$ neurons; gradual: median bias $=-0.36$, $p=0.7$, sign test against 0 followed by HolmBonferroni correction for multiple comparisons, $n=27$ neurons. D, Schematic of experimental protocol to measure two competitor response profiles with 51 stimuli of two different strengths, indicated by magenta and blue dots. $\mathrm{S} 1=11.6 \%$, magenta data; $\mathbf{S} 1=17.6^{\circ} / \mathrm{S}$, blue data. $\boldsymbol{E}, \boldsymbol{F}$, Two competitor response profiles (shown in magenta and blue, respectively) measured using protocol in $\boldsymbol{D}$ at an example neuron with switch-like competitor response profile. $\boldsymbol{E}$, Rasters. $\boldsymbol{F}$, Firing rate plots (conventions are as in Fig. 1). Transition values: $6.95 \%$ (when S1 = 11.6; magenta data) and $12.3^{\circ} / \mathrm{s}$ (when $\mathrm{S} 1=17.6 \%$; blue data). Shift ratio (shift in transition value/change in $S 1$ strength) for this example neuron $=0.88$. $\mathbf{G}-\boldsymbol{I}$, Schematic $(\boldsymbol{G})$ and two competitor response profiles $(\boldsymbol{H}, \boldsymbol{I})$ measured at an example neuron with gradual competitor response profile. Transition values: $3.22 \%$ (when $\mathrm{S} 1=7.6 \%$; green data) and $9.01 \%$ (when $S 1=13.6 \%$; orange data). Shift ratio for this example neuron $=0.96$. $J$, Distribution of the shift ratio across $n=27 \mathrm{Imc}$ neurons. Median shift ratio $=0.89, p=0.26$; sign test against 1. 

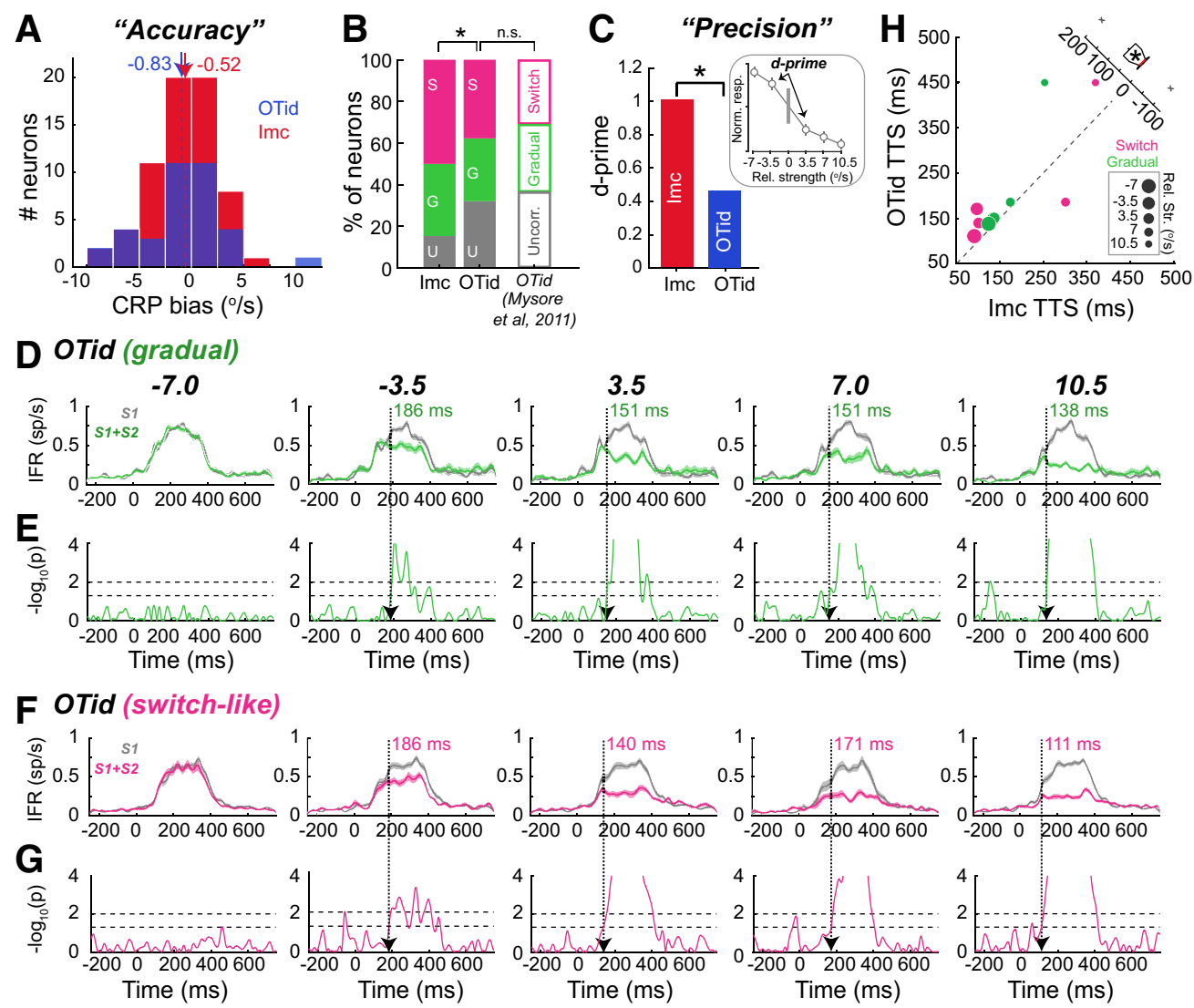

Figure 5. Imc signals stimulus competition with greater precision, and earlier than 0Tid. $A$, Distributions of biases of competitor response profiles of 0 Tid neurons (blue; $n=36$ neurons with correlated competitor response profiles; median $=-0.83 ; p=0.62$; sign test against 0 and Imc neurons (red; data reproduced from Fig. 4B; $n=66 ;$ median $=-0.52$ ). No significant difference between Imc and 0Tid medians ( $p=1.0$, sign test after Holm-Bonferroni correction for multiple comparisons). $\boldsymbol{B}$, Proportions of switch-like (S), gradual (G), and uncorrelated $(U)$ competitor response profiles measured in Imc (stacked bar on left; $n=78$ neurons at which competitor response profiles where measured) and 0Tid (middle bar; $n=53$ neurons at which competitor response profiles were measured). Previously published 0Tid proportions ( $n=169$ neurons at which competitor response profiles were measured; data adapted from Mysore et al., 2011) are also shown for completeness (right bar; unfilled). * (n.s.): $\chi^{2}$ statistic $=5.21, p=0.02(p=1.0), \chi^{2}$ test between Imc and 0Tid proportions measured here followed by Holm-Bonferroni correction for multiple comparisons. C, Comparison of D-prime (Materials and Methods) computed from pooled competitor response profile responses measured in 0Tid (blue) versus $\operatorname{Imc}$ (red). ${ }^{*} p=0.01$, permutation test (Materials and Methods). Inset, Schematic of pooled competitor response profile responses across neurons illustrating D-prime computation across the selection boundary (vertical gray line). D, Pooled instantaneous firing rates (IFRs) of 0Tid neurons with gradual competitor response profiles in response to S1 alone (gray) or paired S1 and S2 (green), binned into five relative strength bins (columns). E, Millisecond-by-millisecond running ANOVA to determine time to suppression (vertical dashed line): the first instant at which responses to paired $S 1$ and $S 2$ diverge significantly from responses to $S 1$ alone (Materials and Methods). OTid responses never diverge significantly for the first relative strength bin (S2-S1 $=-7 \%$; left-most panel). $\boldsymbol{F}, \boldsymbol{G}$, Same as $\boldsymbol{E}$ and $\boldsymbol{F}$, but for 0 Tid neurons with switch-like competitor response profiles (magenta data); OTid responses never diverge significantly for the first relative strength bin (S2-S1 = $-7 \%$ s; left-most column). $\boldsymbol{H}$ Population summary: Scatter plot of time to suppression for OTid neurons versus Imc neurons for the different relative strength bins (i.e., scatter plot of green numbers in $\boldsymbol{D}$ versus magenta numbers in $\boldsymbol{F}$ ). Dots, (Imc, OTid) time to suppression pairs for the different relative strength bins; magenta data, switch-like competitor response profiles; green data, gradual competitor response profiles; progressively larger dots indicate increasing relative strength (S2-S1). For plotting purposes, time to suppression values corresponding to cases in which the responses to paired S1 and S2 never diverged from those to S1 alone, are coded as $450 \mathrm{~ms}$. Dashed line: line of equality. Inset, Boxplot of differences between 0Tid and Imc time to suppression values; ${ }^{*} p=0.02$, sign test against 0 .

boundary (relative strength $=0 \%$; $\mathrm{S} 1=\mathrm{S} 2$; Mysore et al., 2011; Materials and Methods). To compute this metric, we pooled competitor response profile responses across all recorded Imc neurons (gradual, switch-like, and uncorrelated competitor response profiles) binned into five relative strength bins, and then calculated the D-prime between the pooled responses in the relative strength bin of $-3.5 \%$ versus the relative strength bin of $+3.5^{\circ} / \mathrm{s}$ (straddling the boundary; Fig. $5 \mathrm{C}$, inset; Materials and Methods). We repeated this across all OTid neurons. This approach allowed us to estimate the ability of a downstream neuron of Imc (or OTid; ideal observer) to decode the strongest stimulus from population activity in the Imc (OTid). We found that $\mathrm{d}^{\prime}$ across the selection boundary $(\mathrm{S} 1=\mathrm{S} 2)$ was nearly twice as high in the Imc as the OTid (Fig. $5 C$; Imc $=1.02$; OTid $=0.46$; $p=0.01$, permutation test). This result established that the Imc signaled the strongest stimulus more categorically (with greater precision) than the OTid.

Finally, we compared the time course of stimulus competition in the Imc versus the OTid. To this end, we computed the instantaneous firing rates of OTid neurons to S1, and to paired S1 and S2, for each competitor response profile and each strength of S2 (Materials and Methods). Following the procedure used for analyzing Imc time courses, we binned paired S1 $+\mathrm{S} 2$ responses into five bins. Within each bin, we pooled the instantaneous firing rates across switch-like (and separately, across gradual) OTid neurons (Fig. 5D,F; Materials and Methods) and compared the pooled responses to $\mathrm{S} 1$ alone with those of $\mathrm{S} 1+\mathrm{S} 2$ using a millisecond-by-millisecond ANOVA procedure. We quantified separately for OTid neurons with gradual competitor response profiles (Fig. 5E) and switch-like competitor response profiles 
(Fig. 5G) the time to suppression at the different relative strength bins (Fig. 5E,G; Materials and Methods). We found that times to suppression were consistently faster in the Imc than the OTid (Fig. $5 \mathrm{H}$; Imc faster than OT by median value of $18 \mathrm{~ms} ; p=0.02$, sign test).

Together, these quantitative differences in precision and time course of responses to paired S1 and S2 indicate that the Imc is itself a site where computations related to stimulus competition occur, and also that the presence of a competitor is signaled first in the Imc followed by the OTid.

\section{Simultaneous, paired measurements of stimulus competition in the Imc and OTid}

As a final step, we wanted to go beyond comparisons between Imc and OTid populations recorded independently, and directly compare signatures of competition in simultaneously recorded Imc and OT sites encoding for the same portion of sensory space. This approach can reveal how stimulus competition in the two brain areas unfolds at the same time during exposure to the same competing stimuli.

To this end, we first positioned an electrode in the Imc, mapped the RF, and then positioned a second electrode in the OTid such that the OTid RF overlapped with the Imc RF (Fig. $6 A$, spatially "aligned" OTid and Imc RFs, dashed ovals). We simultaneously recorded OTid and Imc responses while presenting S1 and S2 per the competitor response profile stimulus protocol: S1 was presented within the overlapping portion of the RFs, and S2 was presented far outside [ $>30^{\circ}$ away; Fig. $6 A, \mathrm{~S} 1$ and S2 (both were visual looming dots); Materials and Methods].

Responses from a pair of simultaneously recorded, aligned Imc and OTid sites (distance between RF centers $=8^{\circ}$; Materials and Methods) showed that the nature of the competitor response profile was different in the two areas for this example pair (Fig. $6 B, C$, switch-like at the Imc site and gradual at the OTid site). However, both competitor response profiles exhibited negative bias (Fig. 6C, vertical arrows to the left of black arrowheads).

We quantified these properties for each aligned site pair in our population ( $n=26$ pairs) for which both Imc and OTid competitor response profiles were negatively correlated with the strength of S2 ( $n=17$ pairs). Across these 17 pairs of Imc-OTid sites (average difference in centers of $\mathrm{RF}=6.3^{\circ} \pm 0.95^{\circ}$ ), we found that competitor response profile biases in Imc were not different from those in OTid (Fig. $6 D ; p=0.33$, sign test of bias differences between Imc and OTid). Notably, competitor response profile biases at paired Imc and OTid sites were positively correlated (Fig. $6 D$; Pearson's $\rho=0.6, p=0.011$ ). By contrast, competitor response profile transition ranges at paired Imc and OTid sites were uncorrelated (Fig. $6 E$; Pearson's $\rho=-0.37$, $p=0.15$ ). These results regarding competitor response profile biases and transition ranges did not depend on the degree of alignment between the paired Imc and OTid sites (Fig. 6F; bias vs alignment: Pearson's $\rho=0.07, p=0.8$; transition vs alignment: Pearson's $\rho=0.03, p=0.9$ ). Thus, for Imc and OTid sites encoding for the same portion of sensory space, the accuracy of signaling the strongest stimulus was correlated, but precision of the signaling was not.

Finally, we examined the speed at which paired Imc and OTid sites signaled the presence of a competing stimulus. We compared the time course of response suppression by calculating (as before) the time to suppression within each relative strength bin for OTid sites (Fig. 6G,H) as well as paired Imc sites (Fig. 6I, $J)$. The times to suppression at paired Imc and OTid sites were highly correlated (Pearson's $\rho=0.99, p=0.0007$, correlation test), with Imc sites signaling the presence of the competitor earlier than paired OTid, consistent with our findings from independent recordings (Fig. $6 \mathrm{~K}$; best fit line has positive intercept (intercept $=52.8 ; 95 \% \mathrm{CI}, 16.2,89.4$ ), with slope not different from 1 (slope $=0.84 ; 95 \% \mathrm{CI}, 0.66,1.01$ ), indicating that OTid time to suppression are significantly above line of equality].

\section{Discussion}

This study elucidates the properties of multisensory, salience-dependent stimulus competition in a pivotal nucleus in the midbrain selection network in vertebrates, namely the Imc (Graybiel, 1978; Gruberg and Lettvin, 1980; Sereno and Ulinski, 1987; Jiang et al., 1996; Wang et al., 2004). This small group of GABAergic neurons (Wang et al., 2004; Mahajan and Mysore, 2018), which supplies inhibition in a combinatorial manner to all parts of the OT space map (Wang et al., 2004; Mahajan and Mysore, 2018), serves a critical function: without it, competitive interactions and selection in the OTid are abolished (Marín et al., 2007; Mysore and Knudsen, 2013). Considering the critical role of the intermediate and deep layers of the SCid in target selection for spatial attention (McPeek and Keller, 2004; Lovejoy and Krauzlis, 2010), the Imc appears to occupy a spot of central importance within the vertebrate midbrain selection network.

One manner in which the Imc might control competition and selection in the OTid is by serving as a relay of inhibition, converting the input excitatory drive from $\mathrm{OT}_{10}$ into powerful inhibition that is distributed broadly across the OTid space map. Together with the anatomic projection patterns of Imc, this implementation would allow Imc to facilitate computations in the OTid. However, another possibility is that the Imc is itself a site at which computations relating to stimulus competition occur actively (i.e., one at which information about competing stimuli is compared), with this processed information then being relayed to downstream targets (Ipc and OTid). Our results directly support the latter hypothesis.

We found that most Imc neurons $(\sim 85 \%)$ responded to a visual RF stimulus (S1) with decreasing firing rates as the strength of a distant visual competitor (S2) was systematically increased. The responses transitioned from a high to a low value in an abrupt (switch-like) manner in the majority of these cases (60\%), and gradually in the others. Notably, the strength of S2 at which the transition from high to low responses occurred was coupled to the strength of S1, and was, on average, equal to it. These results demonstrated that Imc neurons perform an online comparison of the strengths of the competing stimuli, and signal the strongest one. The large proportion (60\%) of switch-like response profiles resulted in the Imc signaling the strongest stimulus. (We have shown in previous work that a population of neurons in which $\geq 30 \%$ exhibit switch-like competitive response profiles produces categorical signaling at the level of the entire ensemble (Mysore et al., 2011)).

Imc neurons also exhibited multisensory stimulus competition. When Imc was tested with competing stimuli of different sensory modalities, we found results qualitatively similar to those when both stimuli were visual. These results indicated that Imc signals the strongest stimulus, independently of the sensory modalities. Notably, building off of findings that the average transition value of Imc response profiles is equal to the strength of $\mathrm{S} 1$, in the auditory case, the average transition value $(-71 \mathrm{~dB})$ across neurons presents an estimate of the binaural level of an auditory competitor that the Imc deems to be equivalent in strength to the average loom speed of S1 $\left(6.9^{\circ} / \mathrm{s}\right)$. 

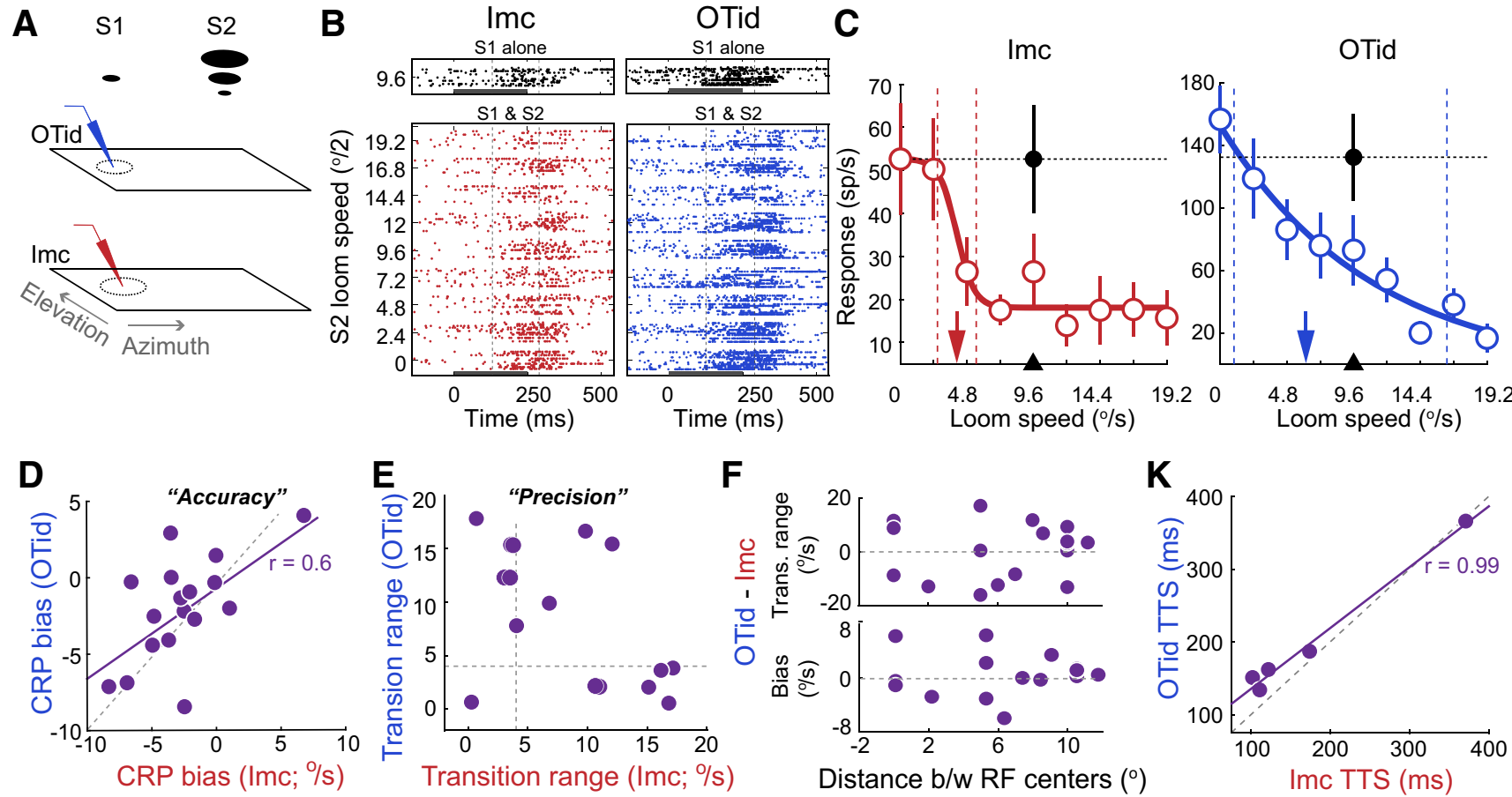

\section{G OTid}

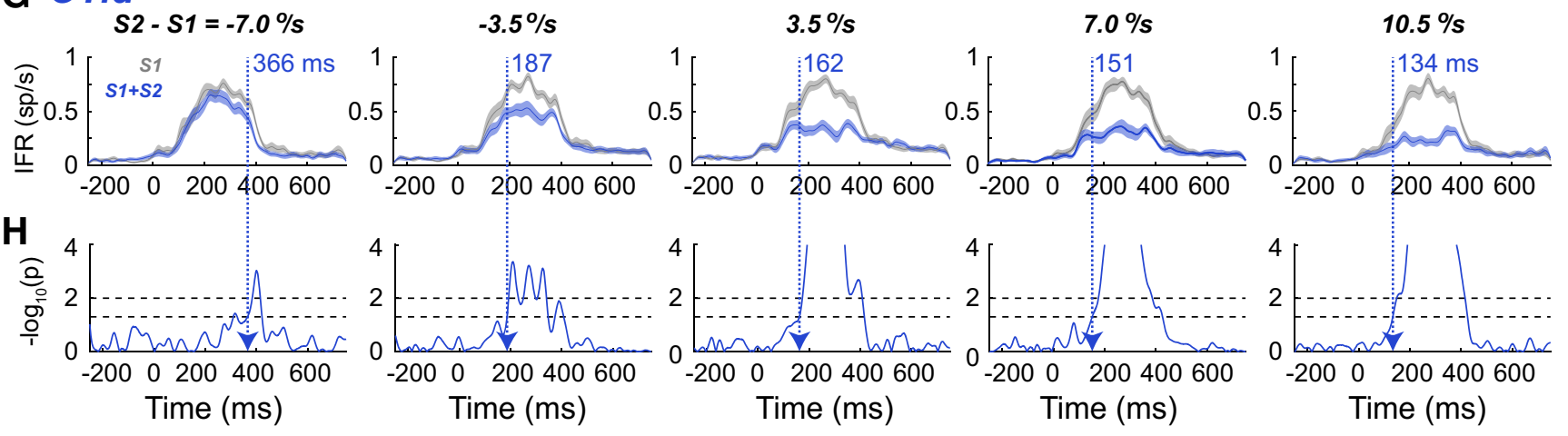

\section{I $I m c_{s 2-s 1=-7.0 \%}$}
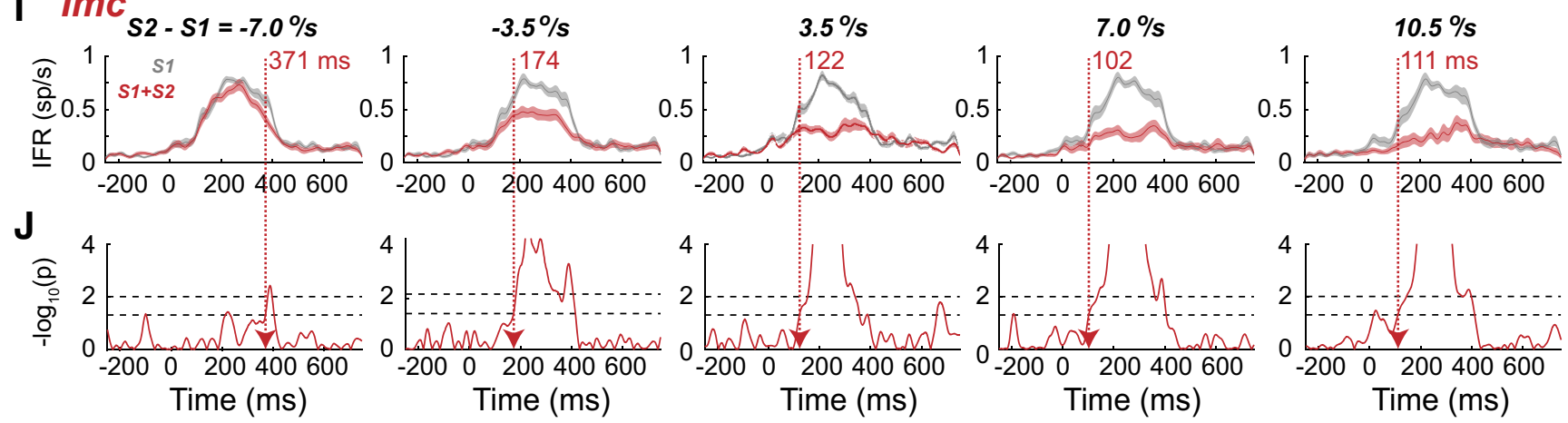

Figure 6. Signatures of stimulus competition at simultaneously recorded, aligned Imc and OTid sites. $\boldsymbol{A}$, Schematic of experimental setup for simultaneous, paired recordings in Imc and OTid. S1 and S2, Stimulus protocol for measuring competitor response profiles; colored icons to the left, recording electrodes, positioned in OTid (blue) and Imc (red); dashed ovals, RFs of recorded site. B, Raster plots of responses for example paired, aligned Imc (left panels) and 0Tid (right panels) sites; distance between RF centers $=8^{\circ}$ (Materials and Methods). Top panels, Responses to $S 1$ alone. Bottom panels, Responses to $S 1$ and $S 2$ presented together. Strength of $S 1=9.6^{\circ} /$. All other conventions similar to Figure $1 B$. $C$, Firing rate responses corresponding to rasters in $\boldsymbol{B}$. Conventions are as in Figure 1 C. Competitor response profile correlation values: $\operatorname{Imc}=-0.81$, $(p=0.009)$; 0Tid $=-0.99, p<0.05(p=0.0000004)$. Competitor response profile transition ranges: $I \mathrm{Imc}=2.8 \%$ (switch-like competitor response profile); $0 \mathrm{Tid}=15.3 \%$ (gradual competitor response profile). Competitor response profile transition values: Imc $=4.1 \% \mathrm{~s}$; 0 Tid $=6.2^{\circ} \%$. D. Scatter plot of competitor response profile biases measured at paired Imc and 0Tid sites $\left(n=17\right.$ pairs; average distance between RF centers $\left.=6.3^{\circ} \pm 0.95^{\circ}\right)$. Dashed gray line, line of equality; solid purple line, best fit line to data; Pearson's $\rho=0.6, p=0.01$. $\boldsymbol{E}$, Scatter plot of transition ranges of competitor response profiles measured at paired Imc and 0Tid sites. Dashed gray lines, cutoff value of transition ranges $(4 \%)$ for switch-like versus gradual competitor response profiles. $\boldsymbol{F}$, Plot of difference in transition ranges of competitor response profiles (OTid - Imc; top) or biases of competitor response profile (0Tid - Imc; bottom) for paired Imc-0Tid sites as a function of distance between Imc and 0Tid RFs. Pearson's $\rho=0.03$, $p=0.9$ (competitor response profile transition ranges); $\rho=0.07, p=0.8$ (competitor response profile biases). $\boldsymbol{G}, \boldsymbol{I}$, Response time courses for 0Tid (G; blue) and Imc sites (I; red) recorded simultaneously; shown are the pooled averages across OTid sites, and separately, across Imc sites (conventions are as in Fig. 2). $\boldsymbol{H}, \boldsymbol{J}$, Results from the millisecond-by-millisecond AVOVA are 
Our results also showed distinct time courses of responses for neurons with switch-like versus gradual competitor response profiles. When S2 was weaker than S1, switch-like neurons signaled the presence of a competitor slower than gradual neurons, but when S2 was stronger than S1, switch-like neurons flipped over to being faster. By contrast, in the OTid, it has been reported that neurons with switch-like profiles are slower to signal the strongest stimulus at all stimulus strengths (Mysore et al., 2011). We found that in the Imc, this potentially puzzling flip was accounted for by differences in the intrinsic nature of switch-like versus gradual neuron response profiles in the Imc, which resulted in greater amounts of response suppression for gradual competitor response profiles when the competitor was weaker than the stimulus in the RF $(\mathrm{S} 2<\mathrm{S} 1)$, but resulted in greater amounts of suppression for switch-like competitor response profiles when the competitor was stronger $(\mathrm{S} 2>\mathrm{S} 1)$. Thus, compared with neurons with gradual competitor response profiles, neurons with switch-like competitor response profiles quickly and effectively signal response suppression when a competing stimulus is stronger. This effect is potentially consistent with circuit mechanisms necessary for producing switch-like response profiles. Recently, it was shown that a "donut-like" pattern of inhibition (Sereno and Ulinski, 1987; Wang et al., 2004) controls the categorical nature of switch-like response profiles by selectively enhancing response differences for stimuli that are on either side of the selection boundary (Mahajan and Mysore, 2020). Such a mechanism could, in principle, account also for the time course effects reported here, although this remains to be tested.

The signatures of competition in the Imc are quantitatively and qualitatively different from those in the OTid. Imc and OTid neurons both signaled the strongest stimulus accurately, with almost no bias in estimating when the two competing stimuli were equal in strength. However, with respect to another key aspect of stimulus competition, namely, the precision with which neurons signal the strongest stimulus-either in a binary-like, explicitly categorical manner, or in a more analog, gradual manner-Imc differed quantitatively from the OTid: it signaled the strongest stimulus much more accurately $(2 \times$ better $)$. These results first came to light from data collected independently (in different experiments) in the Imc and OTid, but using the same experimental setup, stimulus protocols, and analysis pipelines. Subsequently, paired simultaneous recordings in spatially aligned portions of the Imc and OTid not only confirmed these findings, but extended them. They revealed that some aspects of stimulus competition occurred in a coordinated manner in portions of Imc and OTid that encode for the same region of sensory space (i.e., that are active at the same time in a bird experiencing the competing stimuli). Specifically, the bias of neurons in estimating whether a competing stimulus was weaker or stronger than their RF stimulus was highly correlated. This suggests the presence of a shared or mutually dependent mechanism in setting the bias of competition. By contrast, the precision with which neurons signaled the strongest stimulus was not correlated between Imc and

$\leftarrow$

also shown for OTid ( $\boldsymbol{H}$; blue) and Imc sites (J; red), respectively. Columns, Relative strength bins; IFR, instantaneous firing rate. Horizontal dashed lines, $p$ value cutoffs $(0.05$, bottom line; 0.01, top line); vertical dashed arrows (and colored text), time to suppression; Materials and Methods). $\boldsymbol{K}$, Scatter plot of time to suppression measured at aligned Imc versus 0Tid sites recorded simultaneously. Each dot, time to suppression pair for a different relative strength bin; data correspond to the colored numbers in $\mathbf{G}$ and $\boldsymbol{I}$. Dashed line: line of equality. Pearson's $\rho=0.99, p=0.0007$.
OTid neurons encoding for overlapping regions of sensory space. This suggests the presence of independent mechanisms in these two areas involved in setting the precision of competition, providing further support for Imc being an active, independent locus of competition, rather than a simple inhibitory relay.

In interpreting comparisons between functional properties of Imc and OTid neurons, it is important to note that there is much more heterogeneity of cell types (anatomic and molecular) in the OTid than in the Imc. As a result, the observed differences may, in part, be due to nonuniform sampling of the different cell types in the OTid. This concern is somewhat mitigated by two aspects. First, that because the electrodes used in the two areas were identical, any bias in the sizes of the cells recorded is similar in both areas. Second, and perhaps more importantly, the paired ImcOTid recording experiments (Fig. 6) yielded results similar to those of the independent recording experiments (Figs. 1-5). This is pertinent because in the paired recordings, multiunit sites were analyzed (necessarily so, to lawfully define pairs for comparison), whereas in the independent recordings, single units were analyzed. The finding of similar results from both approaches adds confidence to our conclusions.

Analysis of response time courses in both the separate as well as the paired Imc-OTid experiments demonstrated that the Imc reports stimulus competition and signals the strongest stimulus earlier than the OTid. Considering that the Ipc, the other key nucleus in the midbrain selection network, is also a downstream target of the Imc (just as the OTid is), it is plausible that the Ipc will also be slower than the Imc at signaling the strongest stimulus. This hypothesis needs to be tested directly. Consequently, the Imc emerges as the site within the heavily interconnected midbrain selection network at which stimulus competition is potentially resolved first. In any case, the finding above, along with the categorical signaling and anatomic connectivity of Imc, reveals that the Imc sends differentially (categorically) enhanced competitive inhibition to OTid and Ipc sites encoding the weaker versus the stronger competing stimuli.

In summary, not only does the Imc actively perform computations of relative strength-dependent stimulus competition, and does so earlier than the OTid, some aspects of these computations differ qualitatively from paired OTid sites. Overall, the Imc is more categorical in its signaling of the strongest stimulus.

The mechanism by which competition within the Imc may occur is yet to be demonstrated directly. Clues come from modeling and slice experiments, respectively predicting (Mysore and Knudsen, 2012) and demonstrating (Goddard et al., 2014) the presence of long-range inhibitory projections between Imc neurons. These projections could serve to deliver the competitive inhibition (Li et al., 2007) necessary for the functional signatures reported here.

Considering that the midbrain selection network is conserved across vertebrate species (Graybiel, 1978; Gruberg and Udin, 1978; Sereno and Ulinski, 1987; Jiang et al., 1996; Wang et al., 2004), our findings have the power to guide understanding of the midbrain mechanisms underlying spatial selection in other vertebrates. More generally, these findings have implications for unpacking how key computations underlying various forms of selection such as perceptual categorization and value-based decision-making, may be implemented in neural circuits (Mysore and Kothari, 2020). The ubiquity of selection in adaptive behavior, coupled with our current lack of understanding of the precise neural mechanisms that underpin it, highlight the importance of the barn owl midbrain selection 
circuit as a gateway for generating hypotheses about viable circuit solutions for selection and decision-making in general.

\section{References}

Asadollahi A, Knudsen EI (2016) Spatially precise visual gain control mediated by a cholinergic circuit in the midbrain attention network. Nat Commun 7:13472.

Asadollahi A, Mysore SP, Knudsen EI (2010) Stimulus-driven competition in a cholinergic midbrain nucleus. Nat Neurosci 13:889-895.

Asadollahi A, Mysore SP, Knudsen EI (2011) Rules of competitive stimulus selection in a cholinergic isthmic nucleus of the owl midbrain. J Neurosci 31:6088-6097.

David HB (1997) The psychophysics toolbox. Spat Vis 10:433-436.

Duan CA, Erlich JC, Brody CD (2015) Requirement of prefrontal and midbrain regions for rapid executive control of behavior in the rat. Neuron 86:1491-1503.

Dudkin EA, Gruberg ER (2003) Nucleus isthmi enhances calcium influx into optic nerve fiber terminals in Rana pipiens. Brain Res 969:44-52.

Fecteau JH, Munoz DP (2006) Salience, relevance, and firing: a priority map for target selection. Trends Cogn Sci 10:382-390.

Fee MS, Mitra PP, Kleinfeld D (1996) Automatic sorting of multiple unit neuronal signals in the presence of anisotropic and non-Gaussian variability. J Neurosci Methods 69:175-188.

Felsen G, Mainen ZF (2008) Neural substrates of sensory-guided locomotor decisions in the rat superior colliculus. Neuron 60:137-148.

Frost BJ (1978) Moving background patterns alter directionally specific responses of pigeon tectal neurons. Brain Res 151:599-603.

Gabay S, Leibovich T, Ben-Simon A, Henik A, Segev R (2013) Inhibition of return in the archer fish. Nat Commun 4:1657.

Goddard CA, Mysore SP, Bryant AS, Huguenard JR, Knudsen EI (2014) Spatially reciprocal inhibition of inhibition within a stimulus selection network in the avian midbrain. PLoS One 9:e85865.

Graybiel AM (1978) A satellite system of the superior colliculus: the parabigeminal nucleus and its projections to the superficial collicular layers. Brain Res 145:365-374.

Gruberg ER, Lettvin JY (1980) Anatomy and physiology of a binocular system in the frograna pipiens. Brain Res 192:313-325.

Gruberg ER, Udin SB (1978) Topographic projections between the nucleus isthmi and the tectum of the frog Rana pipiens. J Comp Neurol 179:487500 .

Jiang ZD, King AJ, Moore DR (1996) Topographic organization of projection from the parabigeminal nucleus to the superior colliculus in the ferret revealed with fluorescent latex microspheres. Brain Res 743:217-232.

Knudsen EI (1982) Auditory and visual maps of space in the optic tectum of the owl. J Neurosci 2:1177-1194.

Knudsen EI (2011) Control from below: the role of a midbrain network in spatial attention. Eur J Neurosci 33:1961-1972.

Knudsen EI (2018) Neural circuits that mediate selective attention: a comparative perspective. Trends Neurosci 41:789-805.

Krauzlis RJ, Lovejoy LP, Zenon A (2013) Superior colliculus and visual spatial attention. Annu Rev Neurosci 36:165-182.

Li DP, Xiao Q, Wang SR (2007) Feedforward construction of the receptive field and orientation selectivity of visual neurons in the pigeon. Cereb Cortex 17:885-893.

Lovejoy LP, Krauzlis RJ (2010) Inactivation of primate superior colliculus impairs covert selection of signals for perceptual judgments. Nat Neurosci 13:261-266.
Mahajan NR, Mysore SP (2018) Combinatorial neural inhibition for stimulus selection across space. Cell Rep 25:1158-1170.e9.

Mahajan NR, Mysore SP (2020) Neural circuit mechanism for generating categorical representations. bioRxiv 887810. doi:10.1101/2019.12.24.887810.

Marín G, Salas C, Sentis E, Rojas X, Letelier JC, Mpodozis J (2007) A cholinergic gating mechanism controlled by competitive interactions in the optic tectum of the pigeon. J Neurosci 27:8112-8121.

McPeek RM, Keller EL (2002) Saccade target selection in the superior colliculus during a visual search task. J Neurophysiol 88:2019-2034.

McPeek RM, Keller EL (2004) Deficits in saccade target selection after inactivation of superior colliculus. Nat Neurosci 7:757-763.

Mysore SP, Asadollahi A, Knudsen EI (2010) Global inhibition and stimulus competition in the owl optic tectum. J Neurosci 30:1727-1738.

Mysore SP, Asadollahi A, Knudsen EI (2011) Signaling of the strongest stimulus in the owl optic tectum. J Neurosci 31:5186-5196.

Mysore SP, Knudsen EI (2011) Flexible categorization of relative stimulus strength by the optic tectum. J Neurosci 31:7745-7752.

Mysore SP, Knudsen EI (2012) Reciprocal inhibition of inhibition: a circuit motif for flexible categorization in stimulus selection. Neuron 73:193205.

Mysore SP, Knudsen EI (2013) A shared inhibitory circuit for both exogenous and endogenous control of stimulus selection. Nat Neurosci 16:473-478.

Mysore SP, Knudsen EI (2014) Descending control of neural bias and selectivity in a spatial attention network: rules and mechanisms. Neuron $84: 214-226$

Mysore SP, Kothari NB (2020) Mechanisms of competitive selection: A canonical neural circuit framework. eLife, in press.

Pelli DG (1997) The VideoToolbox software for visual psychophysics: transforming numbers into movies. Spat Vis 10:437-442.

Rizzolatti G, Camarda R, Grupp LA, Pisa M (1974) Inhibitory effect of remote visual stimuli on visual responses of cat superior colliculus: spatial and temporal factors. J Neurophysiol 37:1262-1275.

Sasikumar D, Emeric E, Stuphorn V, Connor CE (2018) First-pass processing of value cues in the ventral visual pathway. Curr Biol 28:538-548.e3.

Schellart NAM, Riemslag FCC, Spekreijse H (1979) Center-surround organisation and interactions in receptive fields of goldfish tectal units. Vision Res 19:459-467.

Schryver HM, Mysore SP (2019) Spatial dependence of stimulus competition in the avian nucleus isthmi pars magnocellularis. Brain Behav Evol 93:137-151.

Sereno MI, Ulinski PS (1987) Caudal topographic nucleus isthmi and the rostral nontopographic nucleus isthmi in the turtle, Pseudemys scripta. J Comp Neur 261:319-346.

Shipp S (2004) The brain circuitry of attention. Trends Cogn Sci 8:223-230.

Wang Y, Major DE, Karten HJ (2004) Morphology and connections of nucleus isthmi pars magnocellularis in chicks (Gallus gallus). J Comp Neurol 469:275-297.

Wang Y, Luksch H, Brecha NC, Karten HJ (2006) Columnar projections from the cholinergic nucleus isthmi to the optic tectum in chicks (Gallus gallus): a possible substrate for synchronizing tectal channels. J Comp Neur 494:7-35.

Wang YC, Frost BJ (1991) Visual response characteristics of neurons in the nucleus isthmi magnocellularis and nucleus isthmi parvocellularis of pigeons. Exp Brain Res 87:624-633.

Yan K, Wang SR (1986) Visual responses of neurons in the avian nucleus isthmi. Neurosci Lett 64:340-344. 